\title{
Theoretical Band and Confinement Interpretation of 1D Electrons: Impact on Plasmons and Exchange- Correlation Functionals
}

Akariti Sharma ( $\square$ akariti@prl.res.in )

PRL: Physical Research Laboratory https://orcid.org/0000-0002-9675-8654

\section{Research Article}

Keywords: Confinement, Degeneracy, Exchange- correlation, Plasmons, Effective mass, Spin-orbit splitting

Posted Date: May 3rd, 2021

DOl: https://doi.org/10.21203/rs.3.rs-378654/v1

License: (c) (i) This work is licensed under a Creative Commons Attribution 4.0 International License.

Read Full License 


\title{
Theoretical Band and Confinement Interpretation of 1D Electrons: Impact on Plasmons and Exchange-Correlation Functionals
}

\author{
Akariti Sharma
}

Received: date / Accepted: date

\begin{abstract}
Theoretical band and confinement interpretation of electron gas in lowest energy sub-band of quasi1D metallic wire have been done. Counter effects have been investigated on electrostatic oscillations (plasmons) determined by the electron density response function. Carrier correlations are treated by incorporating the local exchange-correlation $(X C)$ effects within meanfield approximation. Results obtained are in quantitative agreement with experiments data of Nagao et al. (2006 Phys. Rev. Lett. 97 116802). Variation in both degeneracy and confinement potential cause a clear energyshift in the electrostatic oscillations accomplished by asymmetry in band effective mass. Resultant mass asymmetry is attributed to the greater strength of $X C$ effects. These contributions turns out to be quite logical in describing the splitting of 1D-bands over ad-hoc spin-orbital splitting idea of Nagao et al. Calculated $X C$-functionals agreed well with the lattice regularized diffusion Monte Carlo (LRDMC) simulation data (2006 Phys. Rev. B 74, 245427, 2009 J. Phys. A: Math. Theor. 42 214021). Competition among $X C$-functionals and kinetic energy tendencies decides a criterion by satisfying which a metallic quasi-1D wire may undergo an instability at certain critical temperature $\left(T_{c}\right)$.
\end{abstract}

Keywords Confinement · Degeneracy · Exchangecorrelation · Plasmons · Effective mass · Spin-orbit splitting

Akariti Sharma

Theoretical Division, Physical Research Laboratory Ahmedabad,Gujarat,India

Tel.: +91 7009413945

E-mail: akaritisharma@gmail.com

\section{Introduction}

In Physics, Plasmonics relates to the electrostatic oscillations in low-dimensional (LD) metallic systems like nanotubes, nanowires and nanorodes [1-3] which having great technological importance. In particular, the study of plasmons in quasi-1D electrons systems has generated considerable theoretical [4-9] as well as experimental [10-13] interest. The technological progress at nano-scale allows researchers to produce a new class of plasmonic systems called quasi-1D wires having narrow (nm or A) 1D channels. Example includes, confined 1D electron densities at the interface of $\mathrm{AlGaAs}-\mathrm{GaAs}$ hetero-structures [10,14] and array of Au-induced 1D chains on $S i(111)$ surfaces $[11,12]$. Schematically, the $2 \mathrm{D}$ electron gas (2DEG) beneath the gates is repelled

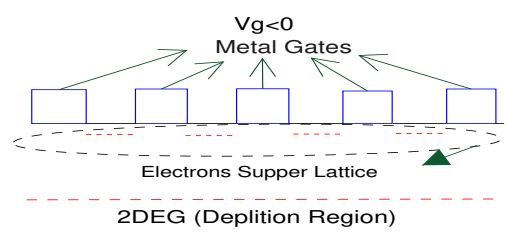

(a)

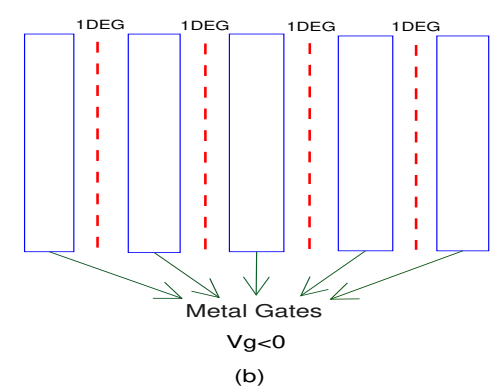

Fig. 1 A set of gates used to form 1D electrons super lattice, lateral view [in panel (a)] and top view [in panel (b)]. 
by negative voltage on the gates and construct a supper lattice of $1 \mathrm{D}$ electrons gas (1DEG) shown in figure 1. So far, Au chains grown on $S i(557)-A u$ surface have attracted much attention due to presence of many intriguing phenomenon such as spin-charge separation [15], Peierls instability, metal-to-insulation transition (MIT) [20], spin-orbital coupling (SOC)[16] etc. due to increased $X C$-effects. The existence of enhanced $X C$ effects is frequently associated with non-local dynamics of carriers [7]. However, the existence of non-local dynamics is not a necessary condition for the increased $X C$-effects. Interestingly, the band and confinement interpretation can also enhance the $X C$-effects in LD systems [19].

Angle-resolved photoemission (ARP) showed that $S i(557)-A u$ surface possess two proximal electron bands and the Fermi-level crossings of these two bands are separated [15]. As a result of which $S i(557)-A u$ surface is metallic despite of having an even electron count. According to ab-initio studies [16], 1D band associated with the $S i(557)-A u$ surface develops appreciable splitting of $(\approx 0.3 \mathrm{eV})$ as consequence of spin-orbit (SO) coupling, and both the bands are metallic. On the other hand, Ahn et al. [17] have found that only one splitted-band is metallic at $T=300 K$ and other is insulating at finite- $T$ close to absolute zero. They also claimed that at least one of the two splitted-bands should be associated with the step-edge atoms. A step head, Alt et al. [18] have found that these two bands associates with two nearly degenerate atomic chains, or a chain of step atoms with two broken bonds. Further, Nagao et al. [11,12] have measured the electrostatic oscillations in the Au chain on the Si(557)-Au surface by using electron-energy-loss spectroscopy (EELS). They noted that Au chain/wire is found to exhibit 1Dmetallic characteristics in one of the SO-splitted band. In spite of these efforts, the surface electronic structure of $S i(557)-A u$ system is not completely understood up to now. Also, the exact nature of $1 \mathrm{D}$-bands and cause of splitting is unclear.

Motivated by these studies, the cause, nature and association of splitted 1D-bands are discussed here on the basis of $X C$-functional in a deliberate manner. Theoretically, the effects of confinement potential $[4,19]$ and degeneracy $[23,24]$ are studied on electron-electron interactions and plasmons, by knowing the fact [1] that plasmons gives reasonably accurate information about the nature and structure of the material through which they propagate. Calculated plasmons are directly compared directly with the experimental findings [11]. Also, the $X C$ - functionals are compared with the available LRDMC [21,22] data.
In section 2, wire model and details of confinement potential models are given. Theoretical method is presented in section 3 , results and discussion are in section 4 followed by conclusion in section 5 .

\section{Wire Model}

Here, the single band model is considered. The Hamiltonian for such a system in second-quantization takes the form as $[23,24]$

$$
\begin{aligned}
H= & \sum_{k \sigma} \epsilon_{k \sigma} a_{k \sigma}^{\dagger} a_{k \sigma} \\
& +\frac{1}{2} \sum_{k_{1}, k_{2}, q, \sigma_{1} \sigma_{2}}\left\langle k_{1}+q, k_{2}+q|V(q)| k_{1}: k_{2}\right\rangle \\
& a_{k_{1}+q, \sigma_{1}}^{\dagger} a_{k_{2}-q, \sigma_{2}}^{\dagger} a_{k_{2} \sigma_{2}} a_{k_{1}} \sigma_{1},
\end{aligned}
$$

where $\epsilon_{k \sigma}=\hbar^{2} k^{2} / 2 m^{*}$ (with band effective mass $m^{*}$ ), $a_{k \sigma}^{\dagger}$ and $a_{k \sigma}$ are creation and annihilation operators. $V(q)$ is the Coulomb interaction potential (electronelectron $(e-e)$ interactions) for wire electrons in $q$ space. In this model, band interpretation is assumed to be done by changing the spin degeneracy factor $g_{s}$. In degenerate state $\left(g_{s}=2\right)$, up-spin and down-spin components are equal while in non-degenerate state $\left(g_{s}=1\right)$ there is an imbalance between up-spin and down-spin components. In quasi-1D wire, Au atoms are modeled as homogeneous gas of electrons embedded in a rigid positive charge background in order to maintain charge neutrality. Electrons are free to move along the length of the wire i.e $x$-axis while their motion is confined quantum mechanically in the $y z$-plane. The electrons are supposes to occupy only the lowest energy sub-band in $y z$-plane due to strong quantum confinement shown schematically in figure 2. Here, parabolic

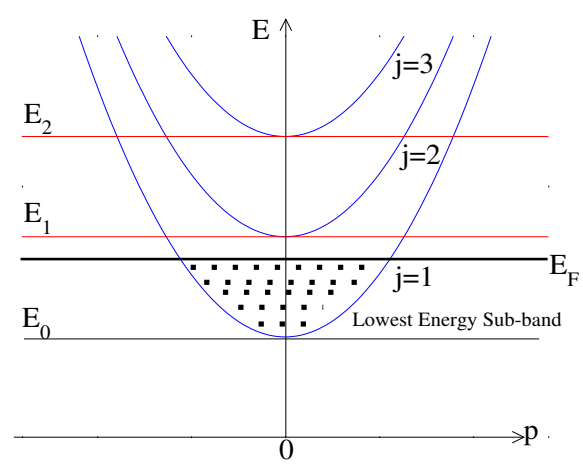

Fig. 2 The total energy $E$ vs the in-layer momentum $p$ of a 2DEG. $E_{F}$ is the Fermi energy, $j$ is the sub-band index, and $E_{1}$ and $E_{2}$ are the discrete energy levels along the quantization direction. Here, only the lowest energy sub-band is shown to be occupied. 
energy sub-band dispersion is considered with an assumption that all complicated band structure effects are represented through the band effective mass $m^{*}$. These assumptions are quite reasonable for $1 \mathrm{D}$ electrons as realized in metallic atomic chains. Therefore, the one-electron wave function in this model is defined as

$$
\Psi_{k}(r)=L^{-1 / 2} e^{\iota k z} \Phi\left(r_{\perp}\right),
$$

where $L$ is the length of the wire and $\Phi\left(r_{\perp}\right)$ is the transverse part of the total wave function with $r_{\perp}$ being the projection of the three dimensional vector $r$ on the transverse plane. Quasi-1D wires corresponds to nonvanishing $\Phi\left(r_{\perp}\right)$, while in clean-1D wires it vanishes. The Coulomb interaction potential in its Fourier transform is defined as

$$
\begin{aligned}
V(q)= & \frac{e^{2}}{\epsilon_{0}} \int_{-\infty}^{\infty} d\left(z-z^{\prime}\right) e^{\iota q\left(z-z^{\prime}\right)} \\
& \int d r_{\perp} d r_{\perp}^{\prime} \frac{\left|\Phi\left(r_{\perp}\right)\right|^{2}\left|\Phi\left(r_{\perp}^{\prime}\right)\right|^{2}}{\left[\left(z-z^{\prime}\right)^{2}+\left(r_{\perp}-r_{\perp}^{\prime}\right)^{2}\right]^{1 / 2}},
\end{aligned}
$$

where $e$ is the electronic charge and $\epsilon_{0}$ is the dielectric constant of the background material.

\subsection{Harmonic Confinement (HC)[4]:}

In the $y z$-plane, the wire electrons are modeled by a symmetric $2 \mathrm{D}$ harmonic potential as

$$
V_{c}\left(r_{\perp}\right)=\hbar^{2} r_{\perp}^{2} /\left(8 m^{*} w^{4}\right),
$$

with $w$ the transverse width of the wire. In the lowest energy sub-band $\Phi\left(r_{\perp}\right)$ takes the form as

$$
\Phi\left(r_{\perp}\right)=\left(2 \pi w^{2}\right)^{1 / 2} e^{\left(R^{2} / 4 w^{2}\right)},
$$

putting the value of $\Phi\left(r_{\perp}\right)$ in the integral of Eq. (3), $V(q)$ can be evaluated as

$$
V(q)=\frac{e^{2}}{\epsilon_{0}} E_{1}\left(q^{2} w^{2}\right) \exp \left(q^{2} w^{2}\right),
$$

with $E_{1}(q w)$ being the exponential-integral function.

\subsection{Infinite Square Well Confinement (ISWC)[19]:}

In the $y z$-plane, $V(q)$ is obtained numerically as

$$
\begin{aligned}
V(q)= & \frac{2 e^{2}}{\epsilon_{0}} \int_{0}^{a} \mathrm{~d} y \int_{0}^{a} \mathrm{~d} y^{\prime} \int_{0}^{a} \mathrm{~d} z \int_{0}^{a} \mathrm{~d} z^{\prime}|\phi(y, z)|^{2} \\
& \times\left|\phi\left(y^{\prime}, z^{\prime}\right)\right|^{2} K_{0}\left(q \sqrt{\left(y-y^{\prime}\right)^{2}+\left(z-z^{\prime}\right)^{2}}\right)
\end{aligned}
$$

with

$$
\Phi(y, z)=\left(\frac{2}{a}\right) \sin \left(\frac{\pi y}{a}\right) \sin \left(\frac{\pi z}{a}\right),
$$

where $K_{0}$ is the zeroth-order modified Bessel function of the second kind and $a$ is the width of the square well. In case of intra-wire $e-e$ interactions $V(q)$ takes the form as

$$
V(q)=\frac{2 e^{2}}{\epsilon_{0}} K_{0}(q w)
$$

While in case of inter-wire $e-e$ interactions (although not considered here) the above equation can be modified as

$$
V_{l l^{\prime}}(q)=\frac{2 e^{2}}{\epsilon_{0}} K_{0}^{\prime}\left(q \sqrt{w^{2}+\left(1-\delta_{l l^{\prime}}\right) d^{2}}\right),
$$

here $l$ and $l^{\prime}$ are the wire indices of two parallel wires and $K_{0}^{\prime}(x)$ is the zeroth-order modified Bessel's function of the first kind. It can be predicted here that, at very small $d$ (center-to-center wire separation), $V_{l l^{\prime}}(q)$ can give more accurate information about the nature and splitting of 1D-band (much progress has already been done).

\section{Theoretical Method}

Dielectric formulation has been used in this work, where the calculations of dynamical susceptibility $\chi(q, \omega)$ is important. The poles of $\chi(q, \omega)$ gives the account of electrostatic excitation energy i.e. $\omega_{p}(q)$. However, the many-world nature of the system forbids exact calculation of $\chi(q, \omega)$, therefore, some approximation has to be made. Here, $\chi(q, \omega)$ is determined by employing the random phase approximation (RPA) [5] within the framework of linear response theory and is given as

$$
\chi(q, \omega)=\frac{\chi_{0}(q, \omega)}{1-V(q) \chi_{0}(q, \omega)},
$$

where

$\chi_{0}(q, \omega)=-\frac{2}{\hbar} \int_{-\infty}^{\infty} \frac{d p}{h} \frac{f^{0}(p+\hbar q / 2)-f^{0}(p-\hbar q / 2)}{\omega-p q / m^{*}+\iota \eta}$.

Take $\hbar=1$ and put $(p-q / 2)=k$ in above Eq. we get

$$
\chi_{0}(q, \omega)=-\frac{1}{\pi} \int_{-\infty}^{\infty} d k \frac{f^{0}(k+q)-f^{0}(k)}{\omega-\frac{q}{m^{*}}\left(k+\frac{q}{2}\right)+\iota \eta},
$$

substitute $k+q=k^{\prime}$ in first term of the above expression and after solving with $f^{0}(p)=\Theta\left(k_{F}-k\right)$ (step function at $T=0 K$ ), we get 


$$
\begin{aligned}
\chi_{0}(q, \omega)=\int_{0}^{k_{F}} \frac{d k}{2 \pi}\left[\frac{-2\left(k q / m^{*}-q^{2} / 2 m^{*}\right)}{\omega^{2}-\left(k q / m^{*}-q^{2} / 2 m^{*}\right)^{2}}\right. \\
\left.+\frac{2\left(k q / m^{*}+q^{2} / 2 m^{*}\right)}{\omega^{2}-\left(k q / m^{*}+q^{2} / 2 m^{*}\right)^{2}}\right] .
\end{aligned}
$$

Integrating Eq.(14), we get

$$
\chi_{0}(q, \omega)=\frac{m^{*} g_{s}}{2 \pi q} \ln \left|\frac{\omega^{2}-\omega_{-}^{2}}{\omega^{2}-\omega_{+}^{2}}\right|,
$$

this $\chi_{0}(q, \omega)$ is the Hartree-Fock dynamical susceptibility of non-interacting electrons with

$$
\omega_{ \pm}=\frac{q k_{F}}{m^{*}} \pm \frac{q^{2}}{2 m^{*}}
$$

here $\omega_{ \pm}$corresponds, respectively, to the upper and lower boundary of the (single) electron-hole pair continuum. In the RPA, we address the local correlations between two electrons via bare $V(q)$ which takes in to account full screening in the system. In Eq. (16), $k_{F}(=$ $\left.\pi n / g_{s}\right)$ is the $1 \mathrm{D}$ Fermi wave vector through which $g_{s}$ comes in to picture. Further, the electrostatic excitation energy $\omega_{p}(q)$ is obtained readily from the poles of Eq. (11) as

$$
\begin{gathered}
1-V(q) \chi_{0}(q, \omega)=0 \\
1-V(q)\left(\frac{m^{*} g_{s}}{2 \pi q}\right) \ln \left|\frac{\omega^{2}-\omega_{-}^{2}}{\omega^{2}-\omega_{+}^{2}}\right|=0 \\
\omega_{p}(q)=\sqrt{\frac{\omega_{-}^{2}-\omega_{+}^{2} e^{\mathcal{O}(q)}}{1-e^{\mathcal{O}(q)}}}
\end{gathered}
$$

with

$$
\mathcal{O}(q)=\frac{2 \pi q}{m^{*} g_{s} V(q)}
$$

Next, the fluctuation-dissipation theorem [23] relates the imaginary part of $\chi(q, \omega)$ with static structure factor $S(q)$ as

$$
S(q)=-\frac{1}{\pi n} \int_{0}^{\infty} d \omega \operatorname{Im} \chi(q, \omega),
$$

here, $n$ is the linear electron number density. With the knowledge of $S(q)$, the total energy $E_{T}$ (per particle) of electrons can be calculated as[24]

$$
E_{T}=\frac{P_{F}^{2}}{6 m^{*}}+\int_{0}^{e^{2}} d \lambda \frac{E_{\text {int }}(\lambda)}{\lambda},
$$

here, first term is the non-interacting kinetic energy while the second term gives the account of exchangecorrelation energy $E_{x c}$ with

$$
E_{\text {int }}(\lambda)=\int_{0}^{\infty} \frac{d q}{2 \pi} V(q, \lambda)[s(q, \lambda)-1],
$$

in above, $V(q, \lambda)=\lambda f(q w) /\left(2 \epsilon_{0}\right) . \lambda$ being the coupling constant and $f(q w)$ (see appendix) is the form factor. $E_{x c}$ accounts for the electronic energy other than non-interacting kinetic and electrostatic energy contributions. Further, the correlation energy $E_{c}$ can be obtained by subtracting the exchange energy $E_{x}$ from the second term of Eq.(22). $E_{x}$ can be obtained from Eq. (23) by replacing $S(q, \lambda)$ with the Hartree-Fock structure factor $S_{0}(q)(=q / 2)$ which is $\lambda$ independent. In the next section, the numerical results of $V(q), \omega_{p}(q)$ and $X C$-functionals are shown at $g_{s}=2$ and $g_{s}=1$ with extra effects of confinement potentials on $V(q)$ and $\omega_{p}(q)$.

\section{Results and Discussion}

In this section, the numerical results are presented for wire parameters which are pertinent to the experimentally fabricated wires. Unless otherwise specified, units used are:, $\omega_{p}(q)$ in $E_{F}, q$ in $\AA^{-1}$ and $w$ in $\AA$. Quasi$1 \mathrm{D}$ wire is characterized by the effective density parameter $r_{s}^{*}\left(=1 / 2 n a_{0}^{*}\right) ; a_{0}^{*}$ is the effective Bohr atomic radius. For brevity, it is assumed that system is at $T=0 K$, though experiments are performed at finite temperature $T$. This assumption seems to be quite reasonable, as $k_{B} T / E_{F}$ is of the order of $10^{-2}$ for Au chain at $T=300 K$. Large Fermi velocity corresponding to room $T$ can be achieved by tuning $r_{s}^{*}$ parameter (effective coupling). For experimental point of view, the used RPA gives good agreement with experimental data in high density regime $\left(0<r_{s}^{*}<1\right)$. This section includes, general wire models, experimental comparison and $X C$ functionals as sub-sections.

\subsection{General Wire Models}

The $g_{s}$-dependence of $V(q)$ vs $q$ at $w=4$ and $r_{s}^{*}=0.62$ is shown in figure 3. $V(q)$ is found to have considerable dependence on the degeneracy factor even at $q \rightarrow 0$. For $g_{s}=2$, the slope of the interaction curve is larger than $g_{s}=1$, indicating that the extent of $e-e$ interactions is more in degenerate state where up-spin and downspin components are equal. In figures 4(a) and (b), the variation of $V(q)$ in two different confinement models is shown at $g_{s}=1$ and $g_{s}=2$, respectively. It is clear that $V(q)$ depends strongly on the nature of the confinement potential. The infinite square-well confinement (ISWC) results in somewhat harder $e-e$ interaction as compared to the harmonic confinement (HC) and effect of both is much harder at $g_{s}=2$ (figure $4(\mathrm{~b})$ ). Also, the difference between the results is slightly more at $g_{s}=1$ (figure 4(a)). Significantly, it is observed that $V(q)$ depends effectively on both the confinement models over 


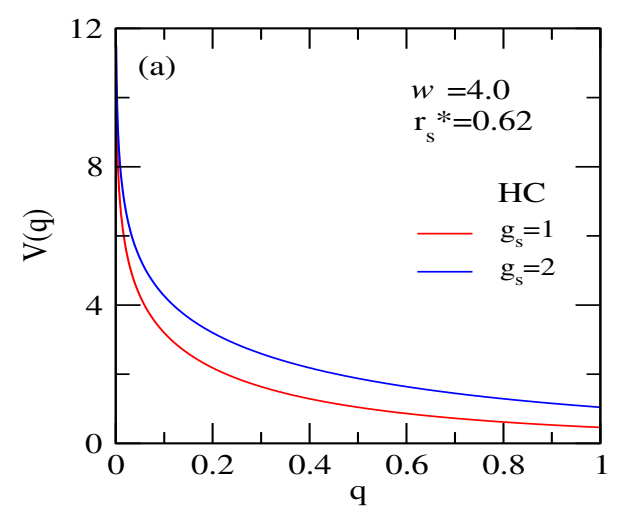

Fig. 3 The variation of $V(q)$ with $q$ at $w=4$ and $r_{s}^{*}=0.62$ for $g_{s}=1$ and $g_{s}=2$ in HC model.

the $q$-range which is accessible to inelastic scattering experiments i.e $(0<q \approx 0.12)$ [11]. The study of band and confinement interpretation of $V(q)$ is worth in order to calculate other exchange-correlational properties of quasi-1D wires in this $q$-range. Here, $q$ is restricted within a proper cut off value $\left(q_{c} \approx 2-3\right)$ to make this model consistent with the assumed single band model $[23,24]$.

To elucidate the role of band and confinement interpretation, the results of $\omega_{p}(q)$ are compared in figure 5 . The numerical values of $\omega_{p}(q)$ are obtained from the
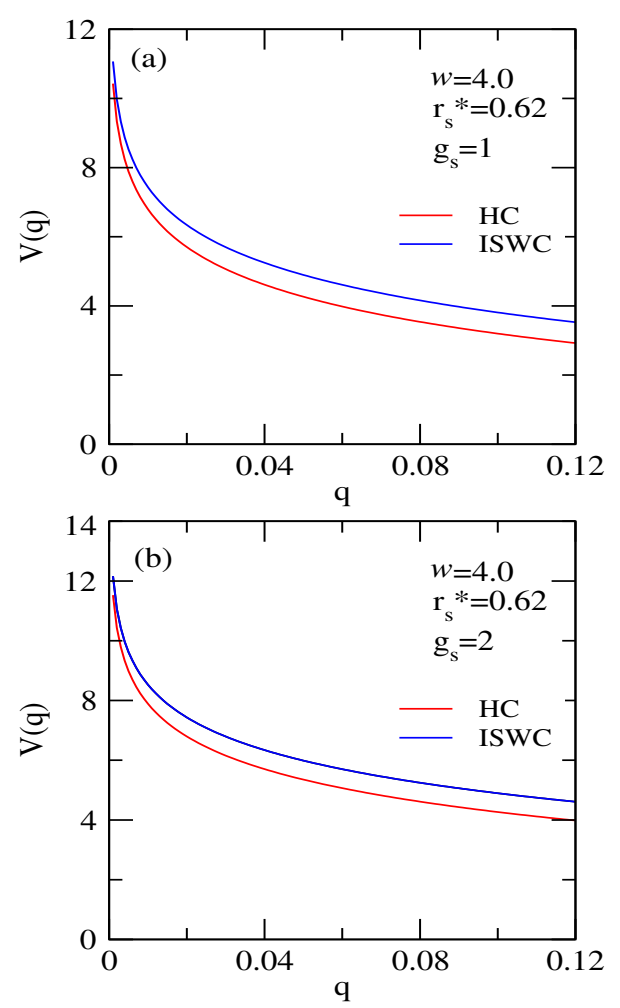

Fig. $4 V(q)$ vs $q$ at $w=4, r_{s}^{*}=0.62$ in $\mathrm{HC}$ and ISWC models for $g_{s}=1$ [in panel (a)] and $g_{s}=2$ [in panel (b)]. poles of $\chi(q, \omega)$ in eq. (11). The harder confinement (ISWC) causes a blue-shift in $\omega_{p}(q)$ w.r.t $\mathrm{HC}$ model in both states, which increases monotonically with $q$. The magnitude of the shift is seen to be slightly increased at $g_{s}=2$ than $g_{s}=1$. This blue-shift in energy is associated with stronger exchange-correlations among electrons under the influence of ISWC. Interestingly, switching from $g_{s}=2$ to 1 cause red-shift in both the models. This indicates the weakening of XC-effects at $g_{s}=1$. Therefore, as an important finding, it is observed that within the bare local potential $V(q)$ (without any non-local corrections), the extent of exchangecorrelations can be modified by doing the band and confinement interpretation of system electrons. In fig-

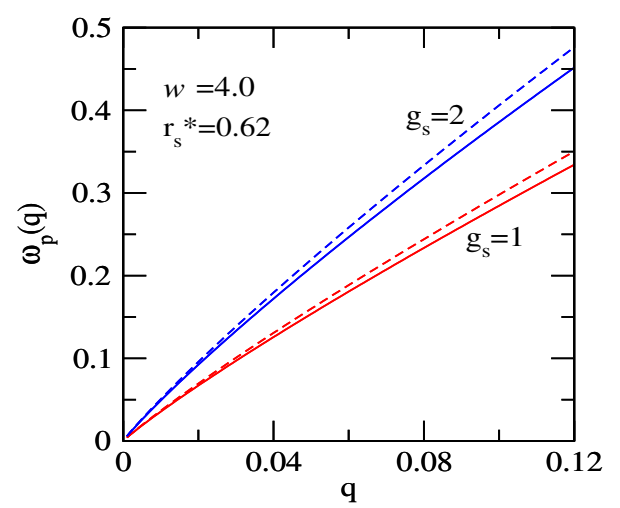

Fig. 5 The variation of $\omega_{p}(q)$ with $q$ at $w=4$ and $r_{s}^{*}=0.62$ for $g_{s}=1$ (red lines) and $g_{s}=2$ (blue lines) in $\mathrm{HC}$ (solid lines) and ISWC (dashed lines) models.

ure $6(\mathrm{a}), r_{s}^{*}$-dependence of $\omega_{p}(q)$ is depicted at fixed width of wire. The energy of $\omega_{p}(q)$ also increases with increase in $r_{s}^{*}$ for both degenerate and non-degenerate states. Increase in energy is attributed to the fact that at high $r_{s}^{*}, e-e$ correlations increases and electrons oscillate collectively with large frequency. Increased $e-e$ correlations bring about more potential energy among system electrons which reflects in their collective excitation spectra i.e $\omega_{p}(q)$ at high $r_{s}^{*}$. It is well checked that, at both (or all) densities, the energy of these oscillations is found to be increased at $g_{s}=2$, but the quantitative effects of degeneracy parameter are more at low densities (high $r_{s}^{*}$ ). In figure 6(b), the wire width dependence of $\omega_{p}(q)$ is shown at fixed $r_{s}^{*}$. Dependence of $w$ is found to be in contrast with $r_{s}^{*}$-dependence. There is loss in energy of $\omega_{p}(q)$ with increase in $w$ at both $g_{s}=1$ and 2. On the lift of degeneracy, energy of $\omega_{p}(q)$ is found to be decreased at both (or all) $w$-values. Therefore, decrease in $w$ and increase in $r_{s}^{*}$ cause qualitatively same effect on $e-e$ interactions irrespective to the confinement model. It is also noted that calculated $\omega_{p}(q)$ at indicated wire parameters lies above the 

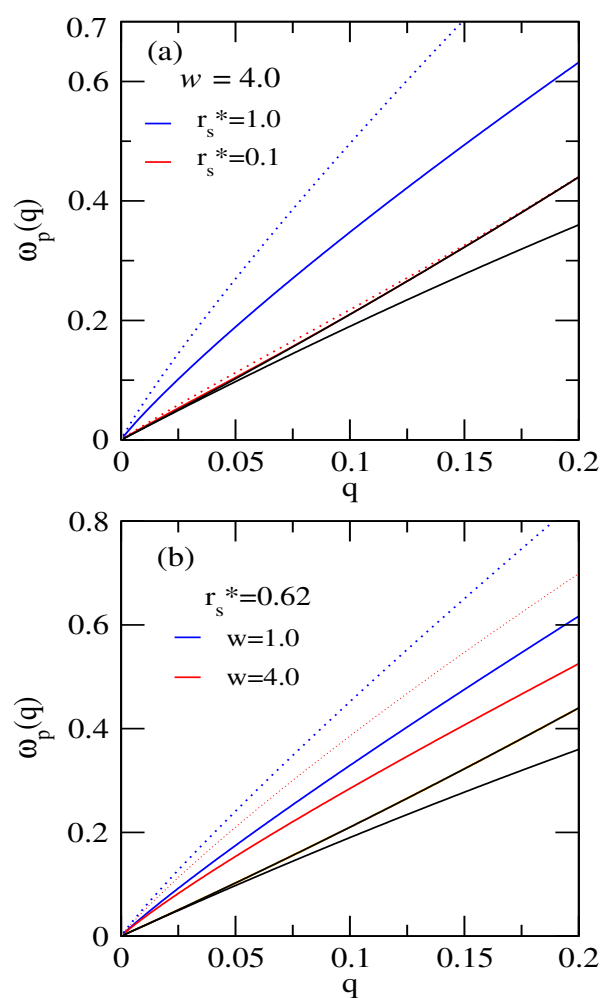

Fig. 6 The dependence of $\omega_{p}(q)$ on $r_{s}^{*}$ [in panel (a)] and $w$ [in panel (b)] at indicated wire parameters for $g_{s}=1$ (solid lines) and $g_{s}=2$ (dotted lines) in $\mathrm{HC}$ model. The thick black lines represent the boundaries of the single electron-hole (eh) pair continuum at $r_{s}^{*}=0.1$ [in panel (a)] and at $w=4.0$ [in panel (b)].

e-h pair continuum and plasmons can be measured experimentally $[7,24]$. For the purpose of generalization of this model, the results of $\omega_{p}(q)$ for an other set of system parameters are plotted in figure 7 along with e-h continuum in ISWC model. At large $w$ and high $r_{s}$, the results obtained are in qualitative agreement with the results shown in figure 6 . For large- $q$ range, it is found that at a certain $q, \omega_{p}(q)$ merges to the single particle e-h pair continuum. It is also noted that, while solving Eq.(11) for $\omega_{p}(q)$, multiple roots have been observed with equal stand probability inside and outside of the e-h pair continuum. Only those values of $\omega_{p}(q)$ are opted which lies above the upper boundary of e-h pair continuum [7]. Here, in both states $g_{s}=1$ and 2 , $\omega_{p}(q)$ lies above the upper boundary of e-h pair continuum. Thus, one can say that metallic behaviour of electrons in single quasi-1D wire is possible in both $g_{s}=1$ and 2 states even at $T=0 K$ which is in contrast to the findings of Ahn et al. [17] observations. At $g_{s}=1$, such metallic behaviour can be completely understood by virtue of the imbalance between spin-components. While in degenerate state, dominating $X C$-effects are responsible for metallic behaviour of electrons. Thus,
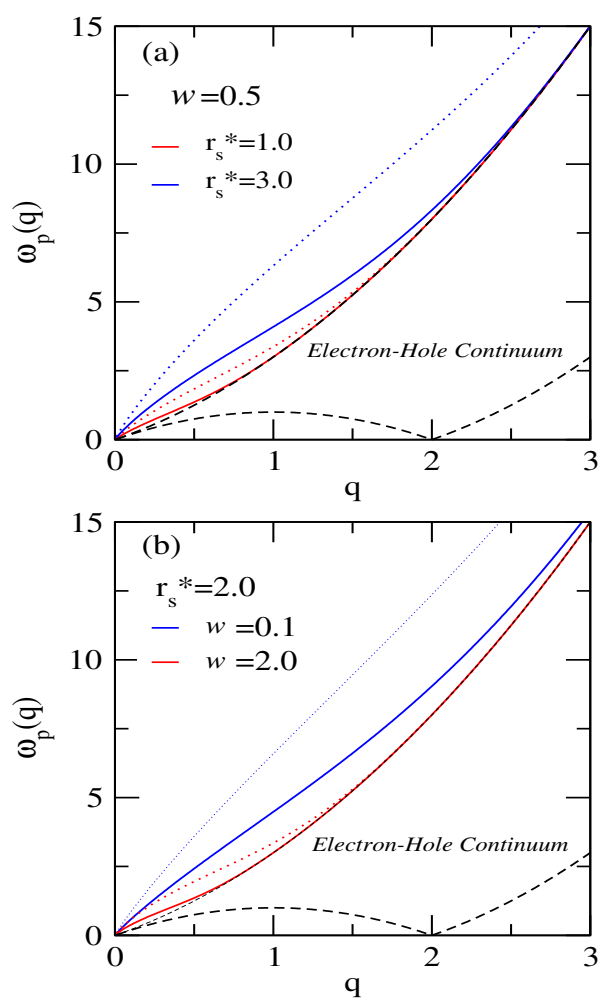

Fig. 7 The dependence of $\omega_{p}(q)$ on $q$ for indicated wire parameters at $g_{s}=1$ (solid lines) and $g_{s}=2$ (dotted lines) in ISWC model. The thick dash-dotted lines represent the boundaries of the single electron-hole (e-h) pair continuum at $r_{s}^{*}=1.0$ [in panel (a)] and at $w=2.0$ [in panel (b)].

the study of $X C$-functionals is very important in order to understand the exact nature of $1 \mathrm{D}$-bands.

\subsection{Experimental Comparison}

To enable a direct comparison with the experimental data [11], the used wire parameters appropriate to the

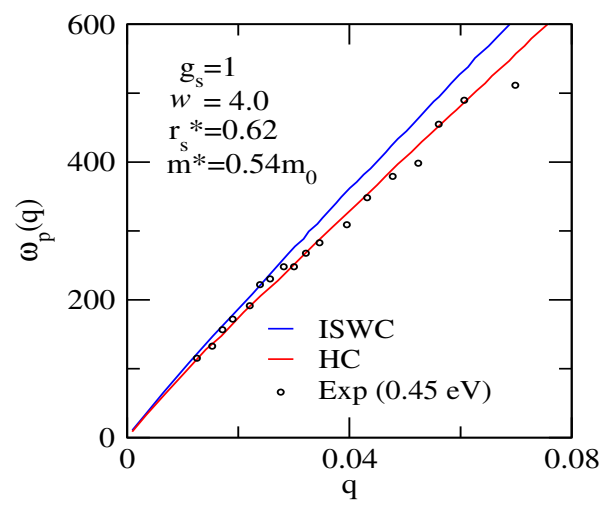

Fig. 8 The variation of $\omega_{p}(q)(\mathrm{meV})$ vs $q$ at indicated wire parameters in HC and ISWC models. The symbols are the experimental data of Nagao et al. [11] for electron beams having incident energy of $45 \mathrm{eV}$. 
$\mathrm{Au}$ chain on the $\mathrm{Si}(557)$ surface are: $\epsilon_{0}=\left(1+\epsilon_{S i}\right) / 2$, with $\epsilon_{S i}=11.5, k_{F}=0.41 \AA^{-1}, w=4 \AA$ and $g_{s}=1$. On experimental lines, only intra-wire interactions are taken in to account by setting degeneracy factor equal to unity. The numerical results of $\omega_{p}(q)$ (in $\mathrm{meV}$ ) vs $q$ are plotted at $w=4, r_{s}^{*}=0.62$ and $m^{*}=0.54 m_{0}$ for both HC and ISWC models in figure 8. In the RPA, by using above wire parameters the $\mathrm{HC}$ model data of $\omega_{p}(q)$ exhibits very good agreement with the experiments data of $\omega_{p}(q)$ obtained by electron-energy-loss spectroscopy (EELS) at incident energy of $45 \mathrm{eV}$. At $g_{s}=1$, reasonably good agreement have been obtained at $m^{*}=0.54 m_{0}$ : a value close to $0.45 m_{0}$ determined by PES [18]; $m_{0}$ is the free electron mass. For these agreed values of $g_{s}$ and $m^{*}$, calculated $r_{s}^{*}=0.62$ and $a_{0}^{*}=11.57 a_{0} ; a_{0}$ is the Bohr atomic radius. This calculated value of $m^{*}$ obtained by fitting the EELS data in the RPA, deviates from the PES results [18] while in close agreement with the theoretical findings of Nagao et al [11]. Conceivably, this departure of $m^{*}$ arise due to the fact that these two experiments (PES and EELS) works in different $k$-states of the band and having different masses of incident particles. In PES, $m^{*}$ reflects the band curvature at low $k$-states near minimum, while in EELS, it reflects the same at higher $k$-states. In EELS experiments, electrostatic oscillations are composed of electronic transitions near the Fermi level and reflect the band dispersion near $E_{F}$. The study of $\omega_{p}(q)$ in this region, requires the incorporation of $X C$-effects on obligatory basis. Here $X C$-effects are already included through RPA, but $m^{*}$ departure is still there which is more in case of ISWC model. It is found that difference in $m^{*}$ is because of the fact that plasmons incorporate the effect of exchange-correlations in addition to the band effective parameters (degeneracy: $g_{s}$ and confinement potential: $V(q))$. By adjusting these parameter a good fit can be obtained between theoretical and experimental data. In Table 1, band fitting parameters of $1 \mathrm{D} \mathrm{Au}$ wire are given in order to see whether a close agreement with data close to $m^{*}=0.45 m_{0}$ can be achieved. The values of $\omega_{p}(q)$ corresponding to $g_{s}=2$ matches the experimental data at a somewhat higher $m^{*}$ i.e $0.57 m_{0}$ even in $\mathrm{HC}$ model. It is also clear from tabulated data that, by choosing a sufficiently larger

Table 1 Band fitting parameters of Au wire at fixed $n$ and $w$.

\begin{tabular}{lcccccc}
\hline Conf. & $\mathrm{n}(\AA)$ & $\mathrm{g}_{s}$ & $\frac{w}{\AA}$ & $r_{s}^{*}$ & $\frac{m^{*}}{m_{0}}$ & $\frac{a_{0}^{*}}{a_{0}}$ \\
\hline $\mathrm{HC}$ & 0.13 & 1 & 4.0 & 0.62 & 0.54 & 11.57 \\
$\mathrm{HC}$ & 0.13 & 2 & 4.0 & 0.33 & 0.57 & 10.96 \\
ISWC & 0.13 & 1 & 4.0 & 0.68 & 0.59 & 10.59 \\
ISWC & 0.13 & 2 & 4.0 & 0.47 & 0.82 & 07.62 \\
\hline
\end{tabular}

$m^{*}, \omega_{p}(q)$ for ISWC model can be brought closer to the experimental data for both $g_{s}=1$ and 2. Therefore, the effective study of plasmons in $1 \mathrm{D}$ wires requires the sound knowledge of band effective parameters along with the technique used to include $X C$-effects. On the other hand, the value of $m^{*}=0.54 m_{0}$ is very close to the theoretically calculated value of $m^{*}=0.52 m_{0}$ by including the effects of non-local corrections (or dynamics of $X C$-effects) [11]. This implies that the proposal of band and confinement interpretation is also capable of incorporating exchange-correlations well within the RPA. Next, the modified single e-h pair continuum

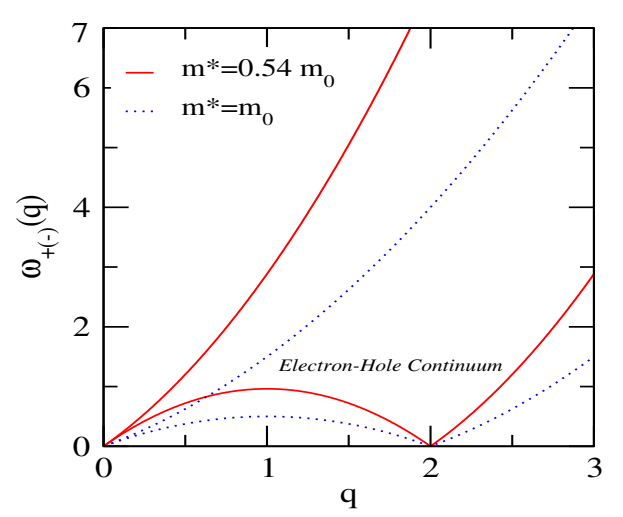

Fig. 9 e-h pair continuum at $m^{*}=0.54 m_{0}$ (red solid lines) and $m^{*}=m_{0}$ (blue dotted lines).

is shown in figure 9, at $m^{*}=0.54 m_{0}$ and $m^{*}=m_{0}$ for $w=4$ and $r_{s}^{*}=0.62$. At $m^{*}=0.54 m_{0}$, the e-h pair continuum oscillates with the higher energy than $m^{*}=m_{0}$. This is due to the fact that, when degeneracy is lifted i.e $g_{s}=1$ electrons shifts to higher $k$-bands leaving behind a hollow positive place at low $k$-bands. Antiparticles (holes) at low $k$-bands having less kinetic energy (velocity) than the electrons at higher $k$-bands. Therefore, one can directly assigned this increased $m^{*}$ to the mass of holes which is responsible for greater $X C$-effects in the system.

\subsection{Exchange-Correlation Functionals}

Although, after having reasonably good agreement with experimental data of Ngao et al., their ad-hoc idea of spin-orbital splitting of 1D-band is not accepted, theoretically. Because they have not proved it rigorously. It is found here that the interplay between band effective parameters and $X C$-functionals can explain splitting of bands in quasi-1D wires in a better way. Because, in order to add spin-orbital coupling parameters in any ab-initio [16] calculations, the exact form of $X C$ functionals is required which helps in formulating the 
$X C$-potential $V_{x c}(r)[25]$ as

$$
V_{x c}(r)=\frac{\delta E_{x c}(r)}{\delta \rho(r)},
$$

here, $E_{x c}$ is the exchange-correlation energy and $\rho(r)$ is the local electronic charge density. The exact value of $E_{x c}$ is difficult to calculate: approximations have to be made at that point. Here, $E_{x c}$ is calculated from Eq. (22) by putting the value of $S(q)$ (from Eq. (21)) in Eq. (23) within the RPA. The numerical results of $E_{x c}$ for $g_{s}=2$ and $g_{s}=1$ of quasi-1D wire are listed in Table 2 , at $w=1$ for some representative values of $r_{s}^{*}$ along with LRDMC data of Casula et al.[21]. It is found that the

Table $2 E_{x c}$ (in Ryd.*/electron) for $w=1$ in RPA along with LRDMC (parentheses) data.

\begin{tabular}{lll}
\hline$r_{s}^{*}$ & $g_{s}=2$ & $g_{s}=1$ \\
\hline 0.2 & -0.751974 & -0.804191 \\
& $(-0.804759)$ & $(-0.804628)$ \\
0.4 & -0.67644302 & -0.751144 \\
& $(-0.675751)$ & $(-0.751468)$ \\
0.6 & -0.62302827 & -0.708838 \\
& $(-0.620978)$ & $(-0.708889)$ \\
0.8 & -0.58325853 & -0.673537 \\
& $(-0.579186)$ & $(-0.673040)$ \\
1.0 & -0.55272631 & -0.643393 \\
& $(-0.546211)$ & $(-0.642101)$ \\
\hline
\end{tabular}

magnitude of $E_{x c}$ increases with increasing $r_{s}^{*}$ in both the spin states i.e., $g_{s}=2$ and $g_{s}=1$. This implies that with increase in $r_{s}^{*}$ (directly proportional to $m^{*}$ ), $E_{x c}$ increases due to enhancement in $m^{*}$. Greater mass of interacting particles give rise to greater $X C$-effects in the system (discussed in sub-section 4.2). Further, the magnitude of $E_{x c}$ is found to be increased at $g_{s}=2$ than $g_{s}=1$ (see table 2) at fixed $r_{s}^{*}$. These findings of $E_{x c}$ are in both qualitative and quantitative agreement with available LRDMC data at $w=1$. For experimentally achievable width of wire $(w=4)$, the numerical results of $E_{x c}$ for $g_{s}=2$ and $g_{s}=1$ are listed in Table 3 and the qualitative behaviour is same as discussed for $w=1$ in Table 2 . The kinetic energy $E_{K . E}$ calculated from first term of Eq.(22) is also shown in figures 10.

Table $3 E_{x c}$ (in Ryd.*/electron) for $w=4$ in the RPA along with LRDMC (parentheses) data.

\begin{tabular}{lll}
\hline$r_{s}^{*}$ & $g_{s}=2$ & $g_{s}=1$ \\
\hline 0.2 & -0.209480 & -0.214308 \\
0.4 & -0.201382 & -0.209433 \\
0.6 & -0.194686 & -0.205107 \\
0.8 & -0.188950 & -0.201203 \\
1.0 & -0.183942 & -0.197628 \\
\hline
\end{tabular}

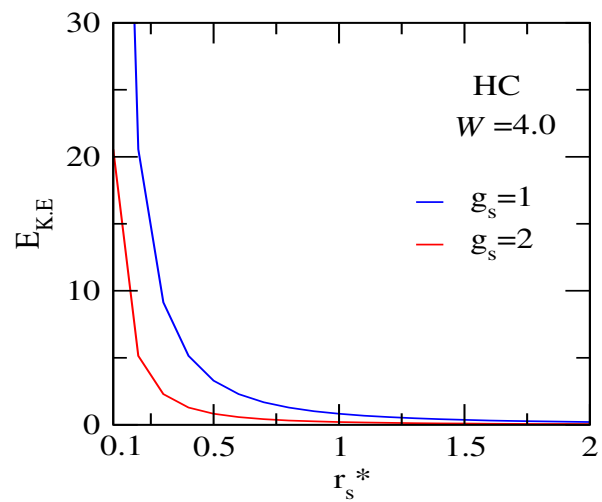

Fig. 10 The $r_{s}^{*}$-dependence of $E_{K . E}$ at $w=4$ in $\mathrm{HC}$ model.

The $r_{s}^{*}$-dependence of $E_{K . E}$ depicts that the kinetic effects are more pronounced at $g_{s}=1$ than $g_{s}=2$. In both situations, the $E_{K . E}$ decreases with increase in $r_{s}^{*}$ and finally becomes zero at sufficiently high value of $r_{s}^{*}$, which is different for $g_{s}=2$ and $g_{s}=1$. At that particular value of $r_{s}^{*}$ (usually high), the $X C$-effects dominates over kinetic effects and as a consequence of which there is a mass-asymmetry among system electrons near Fermi-level. At $E_{F}$, this mass-asymmetry leads to the splitting of 1D-bands and gives a different way to understand band-splitting without ah-hoc $S O$ coupling. Among two splitted 1D-bands, one lies above
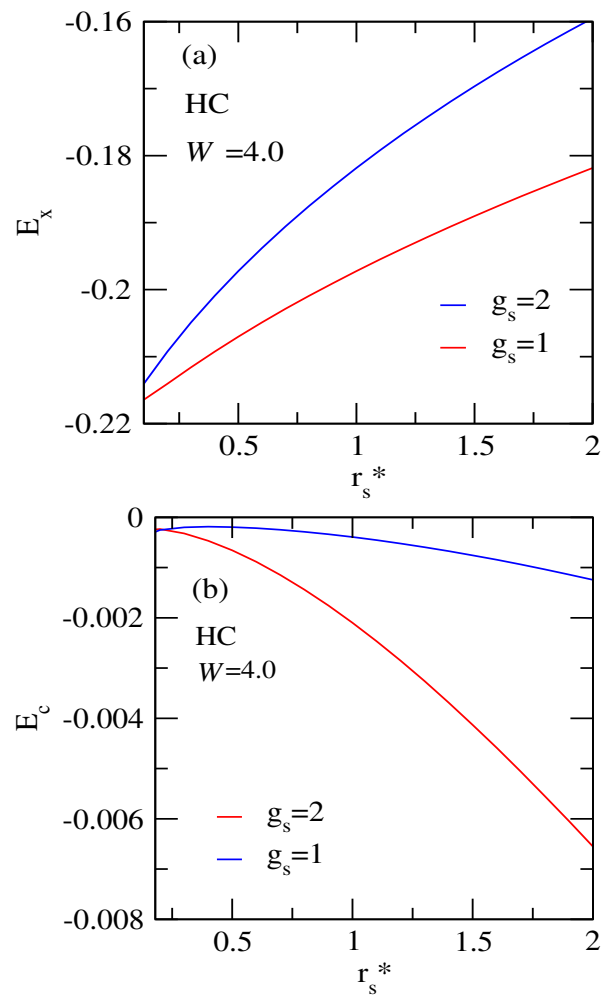

Fig. 11 The $r_{s}^{*}$-dependence of $E_{x}$ [in panel (a)] and $E_{c}$ [in panel (b)] at $w=4$ in $\mathrm{HC}$ model. 
$E_{F}$ and other below $E_{F}$. Theoretically on the basis of band picture (see figure 2), it can be predicted that the electrons in the band below $E_{F}$ are heavier than the electrons in the band above $E_{F}$. Relating these predictions to the assumed intra-wire interactions it is obvious to say that both bands belongs to the same single wire on $S i-A u$ surface. Now, according to this work the observed peaks in experiments [11,15] can also belongs to a single wire. Simultaneously, these peaks can also associates to the different particles in that same wire on the basis of mass-asymmetry among them. Higher peak associates with the lighter electrons at the surface while the lower peak belongs to the heavier electrons at stepedge in the same wire. Therefore, this work supports the experimentally detection of step-edge electrons by Ahn et al.[17] in quasi-1D wires. It is the mass-asymmetry of carrier in the wire which is responsible 1D-band splitting when $X C$-effects are dominant.

Further, the overall behaviour of $X C$-functionals are decided by its two components i.e. $E_{x}$ and $E_{c}$. In figures 11(a) and (b), respectively, the $r_{s}^{*}$-dependence of $E_{x}$ and $E_{c}$ is depicted. At fixed $r_{s}^{*}$, the magnitude of $E_{x}$ is less for $g_{s}=1$ as compared to $g_{s}=2$. On the other hand at $g_{s}=1$, the magnitude of $E_{c}$ is more as compared to $g_{s}=2$. The calculated results of $E_{c}$ are in both quantitative and qualitative agreement with the available LRDMC data of Shulenberger et al. [?] shown in table 4 . Next, in order to study instability

Table $4 E_{c}$ (in Ryd.*/electron) for $w=4$ in RPA along with LRDMC (parentheses) data.

\begin{tabular}{lll}
\hline$r_{s}^{*}$ & $R P A$ & $L R D M C$ \\
\hline 0.2 & -0.000232 & -0.000112 \\
0.4 & -0.000466 & -0.000403 \\
0.6 & -0.000885 & -0.000827 \\
0.8 & -0.001437 & -0.001349 \\
1.0 & -0.002098 & -0.001949 \\
\hline
\end{tabular}

criterion, only one splitted 1D-band is considered with polarization factor: $\zeta=\left(n_{\uparrow}-n_{\downarrow}\right) / n$, where $n_{\uparrow}\left(n_{\downarrow}\right)$ being the density of up (down)-spin components. Here, $\zeta=0\left(g_{s}=2\right)$ and $\zeta=1\left(g_{s}=1\right)$ represent, respectively, the unpolarized and fully-polarized states. Starting from $\zeta=0$ and moving towards $\zeta=1$, the imbalance of spin-components increases, due to which the kinetic energy increases (see figure10) and exchangecorrelation energy decrease (see Table 2). The competition between these two tendencies may leads to charge or spin instabilities in these systems. On the basis of above calculations it is predicted that such instabilities can occurs in quasi-1D wires when following criterion is satisfied by the system electrons

$$
r_{s}^{*}\left[E_{T}(\zeta=1)-E_{T}(\zeta=0)\right]<0,
$$

here, $E_{T}$ is the total energy calculated from Eq.(22). The variation of $E_{T}$ w.r.t $r_{s}^{*}$ at $\zeta=1$ and $\zeta=0$ is shown in figure 12. It is clear from the figure that $E_{T}$

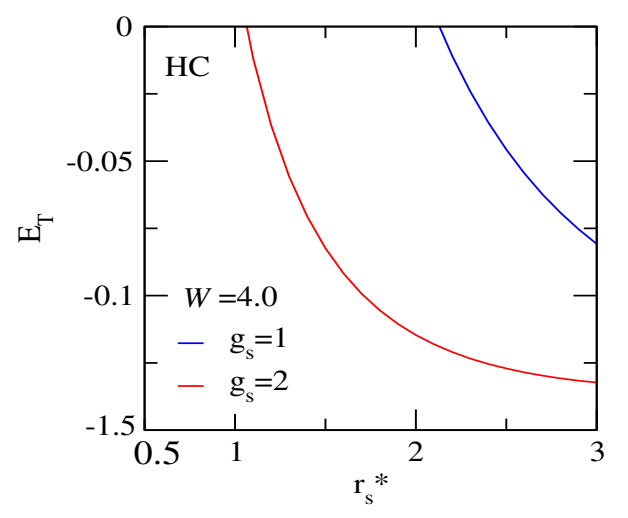

Fig. 12 The $r_{s}^{*}$-dependence of $E_{T}$ at $w=4$ in $\mathrm{HC}$ model.

becomes negative when $X C$-effects dominates over kinetic effects. The negativeness of $E_{T}$ indicates some attractive force among electrons which cause instabilities in these 1D system at particular value of $r_{s}^{*}$. Theoretically, such instabilities can be predicted by calculating the $S(q, \omega)$ as [24]

$$
S(q, \omega)=\frac{\hbar}{\pi} \operatorname{Im} \chi(q, \omega)
$$

The $S(q, \omega)$ describes the density-fluctuation spectrum of the electron system, and can be measured directly through X-ray inelastic scattering experiments. The value of $r_{s}^{*}$ at which the above criterion (Eq. (25)) is satisfied, represented as critical effective electron density $r_{s c}^{*}$. Theoretically, $r_{s c}^{*}$ is difficult to measure in quasi-1D wires and can be further used to calculate critical temperature $\left(T_{c}\right)$. For quasi-1D wires, the fitted formula which relates $r_{s c}^{*}$ to $T_{c}$ of is constructed here as

$$
T_{c}=\frac{T}{T_{F}} ; \quad T_{F}=\frac{\hbar^{2}}{2 m^{*} k_{B}}\left(\frac{\pi}{4 r_{s c}^{*} a_{0}^{*}}\right)^{2} .
$$

The exact value of $T_{c}$ and spin/charge instabilities in quasi-1D wires are still unexplored and demands future investigation. Work related to Eqs. (25), (26) and (27) is in progress as well as the study of plasmons in quasi1D wires are concerned. These findings are important in order to produce increased energy scale Plasmons in these wires by engineering the band structure and confinement potentials. 


\section{Conclusions}

In conclusion, electrons in $\mathrm{HC}$ model serves as a good approximation for theoretical description of the plasmons in atomic scale metal wire at experimentally accessible $r_{s}^{*}$ and $w . V(q)$ and $\omega_{p}(q)$ depends strongly on the confinement model and degeneracy factor. Plasmons are found to relied upon $m^{*}, r_{s}^{*}$ and the nature of the 1D-band. Further, the $X C$-effects are more significant at high $r_{s}^{*}$ and low $w$. However, their influence grows with increasing confinement and imposing degeneracy in the system. These correlations are found to cause a noticeable energy-shift in $\omega_{p}(q)$. This implies that band and confinement interpretation of $1 \mathrm{D}$ electrons may cause enhanced $X C$-effects even at high densities and leads to the phenomenon of mass-asymmetry. The exploration of the competition between exchangecorrelation functionals gives a better idea about 1Dband splitting over the ad-hoc assumption of $S O$-coupling. Based on $X C$-functionals, an instability criterion is proposed, satisfying which a quasi-1D wire may host an (spin/charge) instability at particular $T_{c}$. Simultaneously, one can create $X C$-functionals for quasi-1D wires by mixing and matching given data of exchange and correlation functionals for the purpose of $a b$-initio calculations. Overall these finding may prove useful in fabrication of $1 \mathrm{D}$ plasmonic devices like spin transistors, spin filter etc.

Acknowledgements This work was performed in parts at Physical Research Laboratory (PRL) Ahmedabad and at Punjabi University Patiala in India. The support and resources from PRL equipped with Vikram-100 High Performance Compute Cluster are gratefully acknowledged.

\section{Appendix 1}

In $\mathrm{HC}$, for the calculations of $V(q)$ in Eq.(6), the limiting forms of form factor i.e. $f(q w)=2 E_{1}\left(q^{2} w^{2}\right) \exp \left(q^{2} w^{2}\right)$ are required which can be calculated as

$f(q w)= \begin{cases}\frac{2}{q^{2} w^{2}} \quad \text { for } & (q w \gg 1) \\ 4 \ln \left|\frac{1}{q w}\right| \text { for } & (q w \ll 1)\end{cases}$

In ISWC, for the calculations of $V(q)$ in Eq.(9), the limiting forms of the form factor i.e. $g(q w)$ are required which can be calculated as

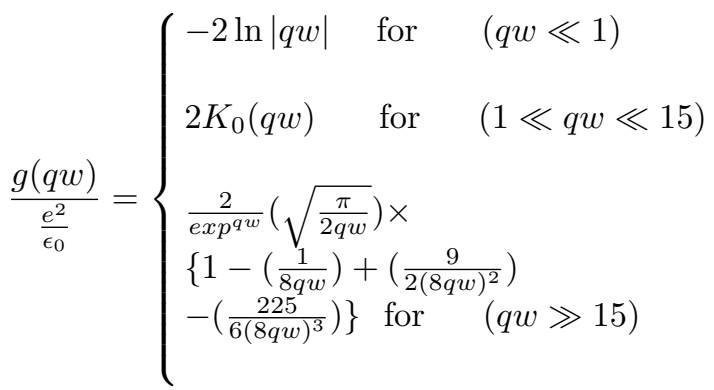

\section{Funding}

This work is funded by Physical Research Laboratory, Ahmedabad supported by Department of Space (DoS), Government of India.

\section{Conflicts of interest/Competing interests}

Author having no conflicts of interest.

\section{Availability of data and materials}

Data and materials available on request from the author.

\section{Code availability}

Code for this work is confidential, but may available on written application which includes the description of needs and collaboration with the author.

\section{Authors' contributions}

The whole project is designed by Akariti Sharma.

\section{Ethics approval}

Not applicable

\section{Consent to participate}

Consent is clear and having permission for related research activities.

\section{Consent to publish}

Consent is clear for the publication of this work. 


\section{References}

1. Ting Zhang, Steve Blair, Gray Level Image Encoding in Plasmonic Metasurfaces, Plasmonics 15: 1305-1311 (2020). https://doi.org/10.1007/s11468-020-01151-5.

2. Gao X, Hui Shi J, Shen X, Feng Ma H, Xiang Jiang W, Li L, Jun Cui T, Ultrathin dual-band surface plasmonic polariton waveguide and frequency splitter in microwave frequencies, Appl Phys Lett 102(15): 151912 (2013). https://doi.org/10.1063/1.4802739.

3. Blaber M G, Arnold M D, Ford M J, A review of the optical properties of alloys and intermetallics for plasmonics, J Phys Condens Matter 22(14): 143201 (2010). https://doi.org/10.1088/0953- 8984/22/14/143201.

4. Friesen W I, Bergersen B, Dielectric response of a onedimensional electron gas, J. Phys. C: Solid State Phys.13, 6627 (1980). https://doi:10.1088/0022-3719/13/36/016.

5. Das Sarma S, Lai W-Y, Screening and elementary excitations in narrow-channel semiconductor microstructures, Phys. Rev. B 32, 1401 (1985). https://doi.org/10.1103/PhysRevB.32.1401.

6. Gold A, Ghazali A, Analytical results for semiconductor quantum-well wire: Plasmons, shallow impurity states, and mobility Phys. Rev. B 41, 7626 (1990). https://doi.org/10.1103/PhysRevB.41.7626.

7. Das Sarma S, Hwang E H, Dynamical response of a one-dimensional quantum-wire electron system, Phys. Rev. B 54, 1936 (1996). https://doi.org/10.1103/PhysRevB.54.1936.

8. Afshin Moradi, Plasmon modes of metallic nanowires including quantum nonlocal effects, PHYSICS OF PLASMAS 22, 032112 (2015). http://dx.doi.org/10.1063/1.4916059.

9. S. Ali, Shafiq Ur Rehman, Z. J. Ding, Stable plasmon excitations in quantum nanowires, PHYSICS OF PLASMAS 25, 082115 (2018).https://doi.org/10.1063/1.5041297.

10. Goñi A R, Pinczuk A, Weiner J S, Calleja J M, Dennis B S, Pfeiffer L N, West K W, One-dimensional plasmon dispersion and dispersionless intersubband excitations in GaAs quantum wires, Phys. Rev. Lett. 67, 3298 (1991). https://doi.org/10.1103/PhysRevLett.67.3298.

11. Nagao $\mathrm{T}$, Yaginuma $\mathrm{S}$, Inaoka $\mathrm{T}$, Sakurai $\mathrm{T}$, One-Dimensional Plasmon in an AtomicScale Metal Wire, Phys. Rev.Lett. 97, 116802 (2006).https://doi.org/10.1103/PhysRevLett.97.116802.

12. Nagao $\mathrm{T}$, Yaginuma $\mathrm{S}$, Inaoka $\mathrm{T}$, Sakurai $\mathrm{T}$, Jeon $\mathrm{D}$, Sound-Wave-Like Collective Electronic Excitations in $\mathrm{Au}$ Atom Chains, J. Phys. Soc. Japan 76, 114714 (2007). https://doi.org/10.1143/JPSJ.76.114714.

13. Liu C, Inaoka $\mathrm{T}$, Yaginuma $\mathrm{S}$, Nakayama $\mathrm{T}$, Aono $\mathrm{M}$, Nagao T, Disappearance of the quasi-one-dimensional plasmon at the metal-insulator phase transition of indium atomic wires, Phys. Rev. B 77, 205415 (2008). https://doi.org/10.1103/PhysRevB.77.205415.

14. Thornton T J, Pepper M, Ahmed H, Andrews D, Davies G J, One-Dimensional Conduction in the 2D Electron Gas of a GaAs-AlGaAs Heterojunction, Phys. Rev. Lett. 56, 1198 (1986) https://doi.org/10.1103/PhysRevLett.56.1198, and Berggren K-F, Thornton T J, Newson D J, Pepper M, Magnetic Depopulation of 1D Subbands in a Narrow 2D Electron Gas in a GaAs:AlGaAs Heterojunction, Phys. Rev. Lett. 57, 1769 (1986). https://doi.org/10.1103/PhysRevLett.57.1769.

15. Losio R, Altmann K N, Kirakosian A, Lin J-L, Petrovykh D Y, Himpsel F J, Band splitting for $\mathrm{Si}(557)$-Au: is it spin-charge separation?, Phys. Rev. Lett. 86, 4632 (2001). https://doi.org/10.1103/PhysRevLett.86.4632.
16. Sánchez-Portal D, Riikonen S, Martin R M, Role of Spin-Orbit Splitting and Dynamical Fluctuations in the Si(557)-Au Surface, Phys. Rev. Lett. 93, 146803 (2004). https://doi.org/10.1103/PhysRevLett.93.146803.

17. Ahn J R, Yeom H W, Yoon H S, Lyo I-W, MetalInsulator Transition in $\mathrm{Au}$ Atomic Chains on $\mathrm{Si}$ with Two Proximal Bands, Phys. Rev. Lett. 91, 196403 (2003). https://doi.org/10.1103/PhysRevLett.91.196403.

18. Altmann K N, Crain J N, Kirakosian A, Lin J-L, Petrovykh D Y, Himpsel F J, Losio R, Low-dimensional electronic states at silicon surfaces, Phys. Rev. B 64, 035406 (2001).https://doi.org/doi:10.1007/s00339-005-3365-3.

19. Campos V B, Degani M H, Hipólito O,Exchange and Correlation-Effects in a Semiconductor QuantumWell Wire, Superlatt. Microstruct. 17, 85 (1995). https://doi.org/10.1006/spmi.1995.1018.

20. Robinson I K, Bennett P A, Himpsel F J, Structure of Quantum Wires in $\mathrm{Au} / \mathrm{Si}(557)$, Phys. Rev. Lett. 88, 096104 (2002). https://doi.org/10.1103/PhysRevLett.88.096104.

21. Michele Casula, Sandro Sorella, Gaetano Senatore, Ground state properties of the one-dimensional Coulomb gas using the lattice regularized diffusion Monte Carlo method, Phys. Rev. B 74, 245427 (2006). https://doi.org/10.1103/PhysRevB.74.245427.

22. Luke Shulenburger, Michele Casula, Gaetano Senatore, and Richard M. Martin, Spin resolved energy parametrization of aquasi-one-dimensional electron gas, J. Phys. A: Math. Theor. 42, 214021 (2008). https://iopscience.iop.org/article/10.1088/17518113/42/21/214021/pdf.

23. D. Pines, P. Nozières, The Theory of Quantum Liquids. Benjamin, New York (1966). ISBN-13: 9780201407747,ISBN-10: 0874809355.

24. See, for example, G. D. Mahan, Many Particle Physics. Plenum Press, New York (1993). ISBN 978-1-4757-5714-9.

25. Kun Cao, Henry Lambert, Paolo G. Radaelli, Feliciano Giustino, $\mathrm{Ab}$ initio calculation of spin fluctuation spectra using time-dependent density functional perturbation theory, plane waves, and pseudopotentials, Phys. Rev. B 97, 024420 (2018). https://doi.org/10.1103/PhysRevB.97.024420 
Figures

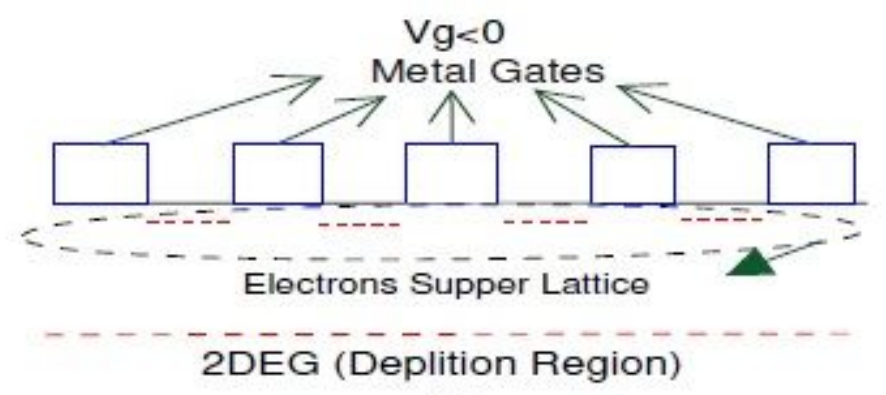

(a)

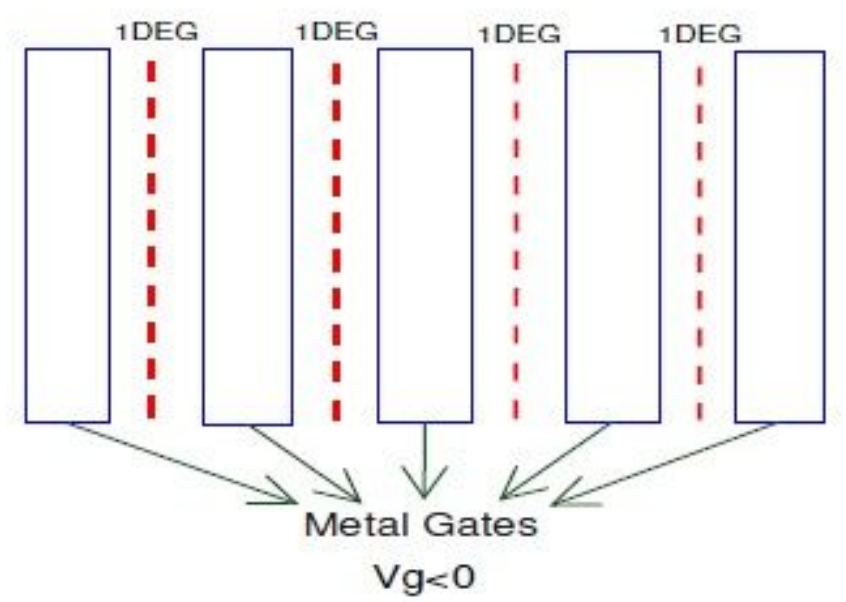

(b)

\section{Figure 1}

A set of gates used to form 1D electrons super lattice, lateral view [in panel (a)] and top view [in panel (b)]. 


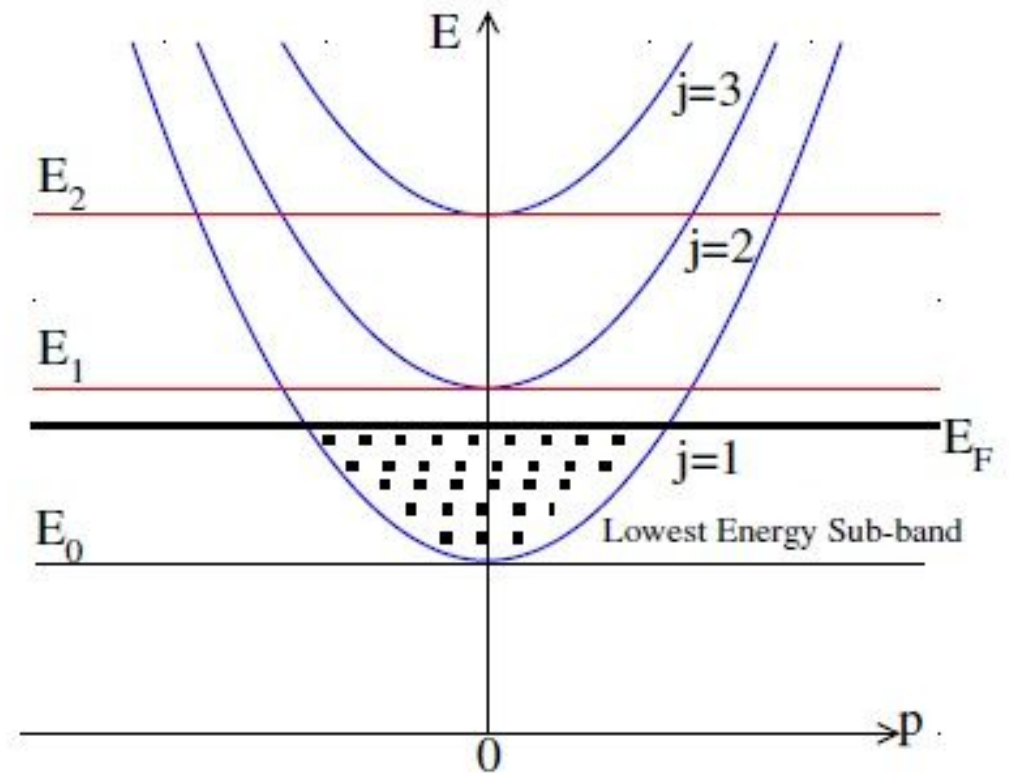

Figure 2

The total energy $E$ vs the in-layer momentum $p$ of a 2DEG. EF is the Fermi energy, $j$ is the sub-band index, and $\mathrm{E} 1$ and $\mathrm{E} 2$ are the discrete energy levels along the quantization direction. Here, only the lowest energy sub-band is shown to be occupied.

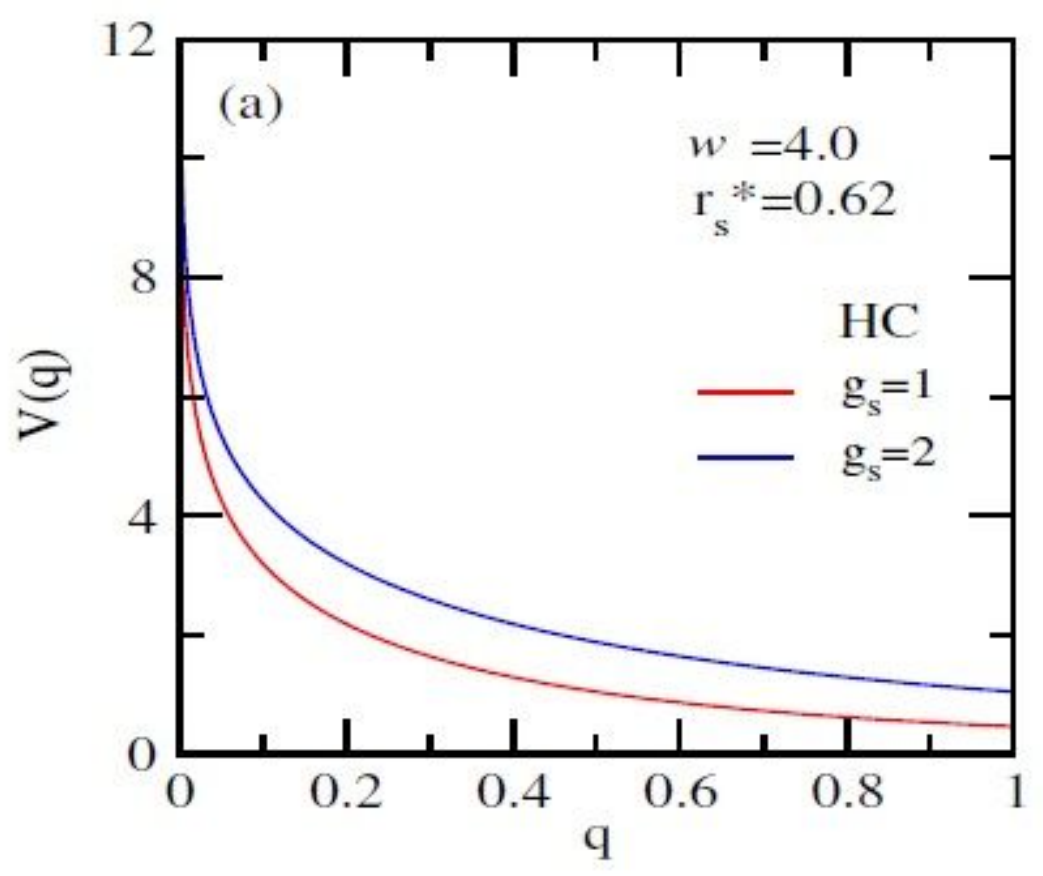

\section{Figure 3}

The variation of $\mathrm{V}(\mathrm{q})$ with $\mathrm{q}$ at $\mathrm{w}=4$ and $\mathrm{r}^{*} \mathrm{~s}=0: 62$ for $\mathrm{gs}=1$ and $\mathrm{gs}=2$ in $\mathrm{HC}$ model. 

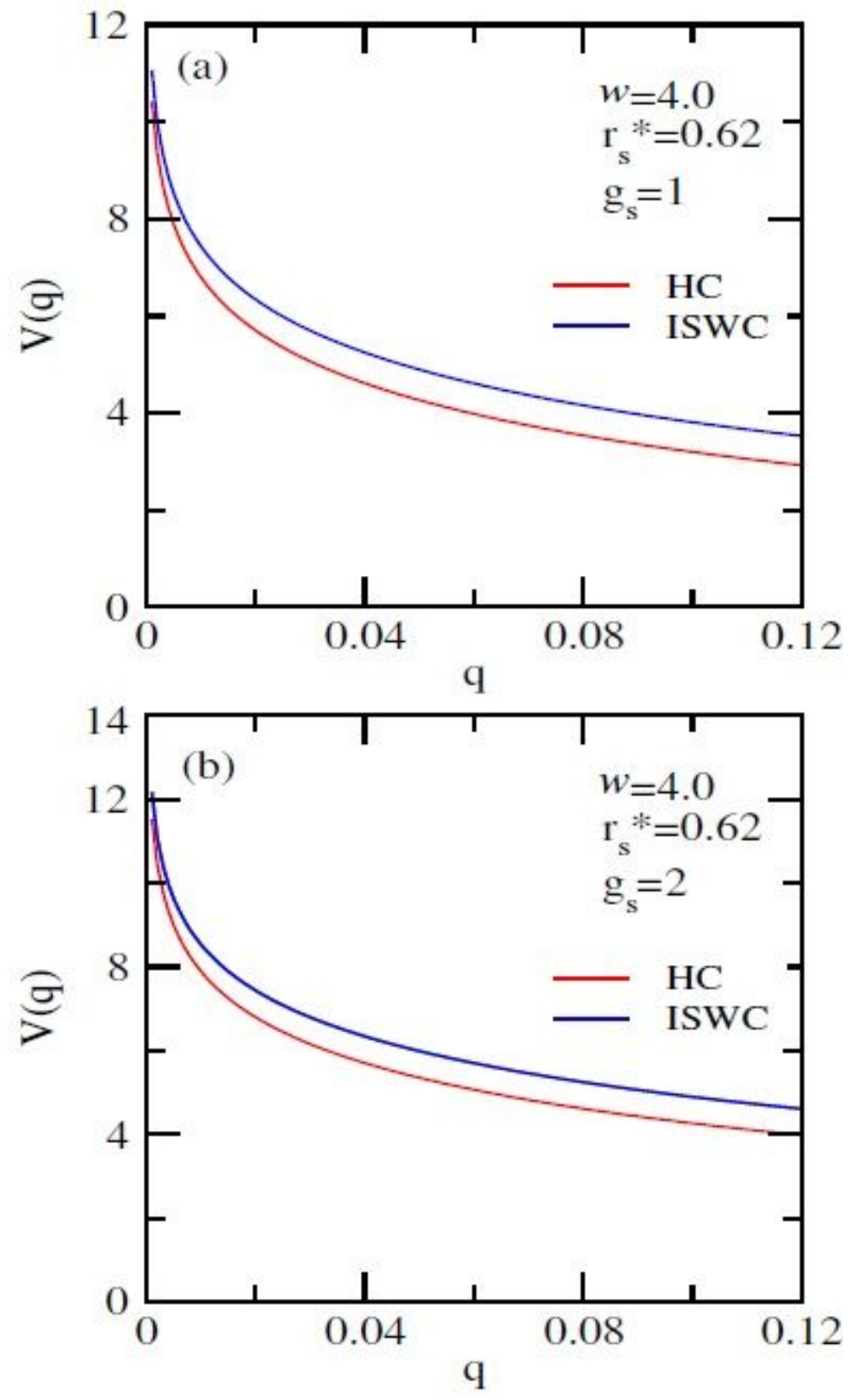

Figure 4

V (q) vs q at $w=4, r^{\star} s=0: 62$ in HC and ISWC models for gs $=1$ [in panel (a)] and gs $=2$ [in panel (b)]. 


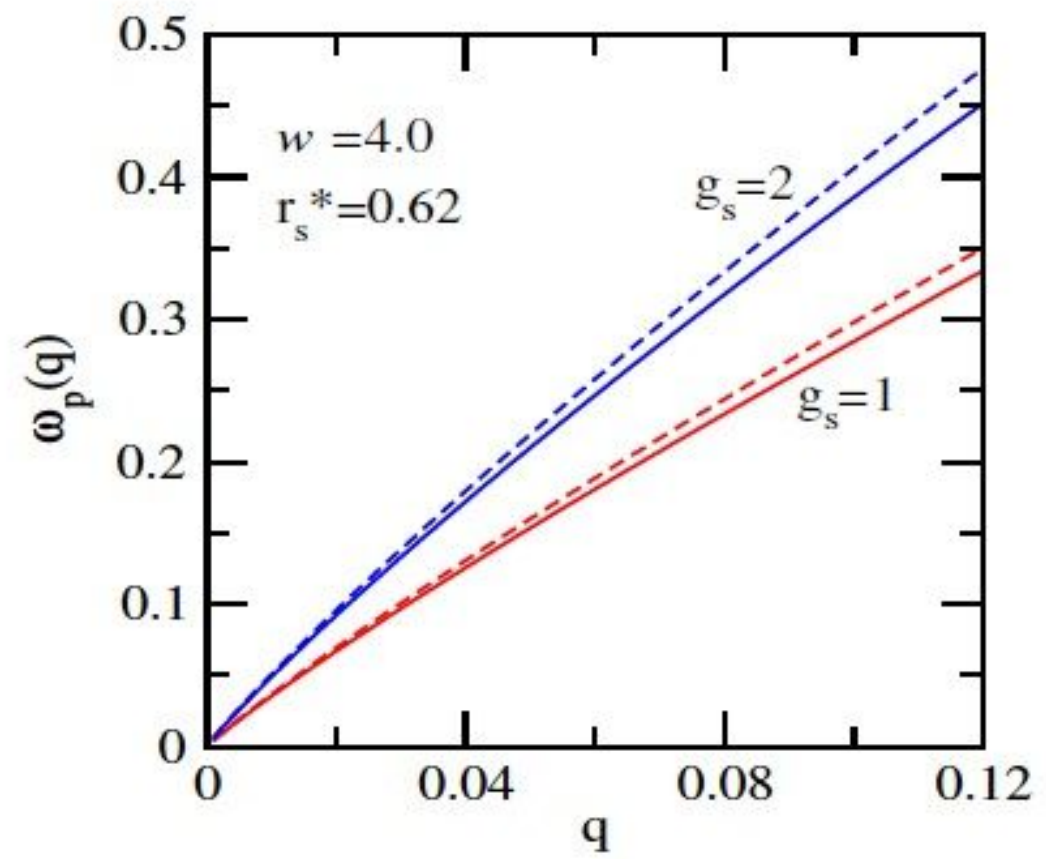

Figure 5

The variation of $! p(q)$ with $q$ at $w=4$ and $r^{\star} s=0: 62$ for $g s=1$ (red lines) and gs $=2$ (blue lines) in $\mathrm{HC}$ (solid lines) and ISWC (dashed lines) models. 

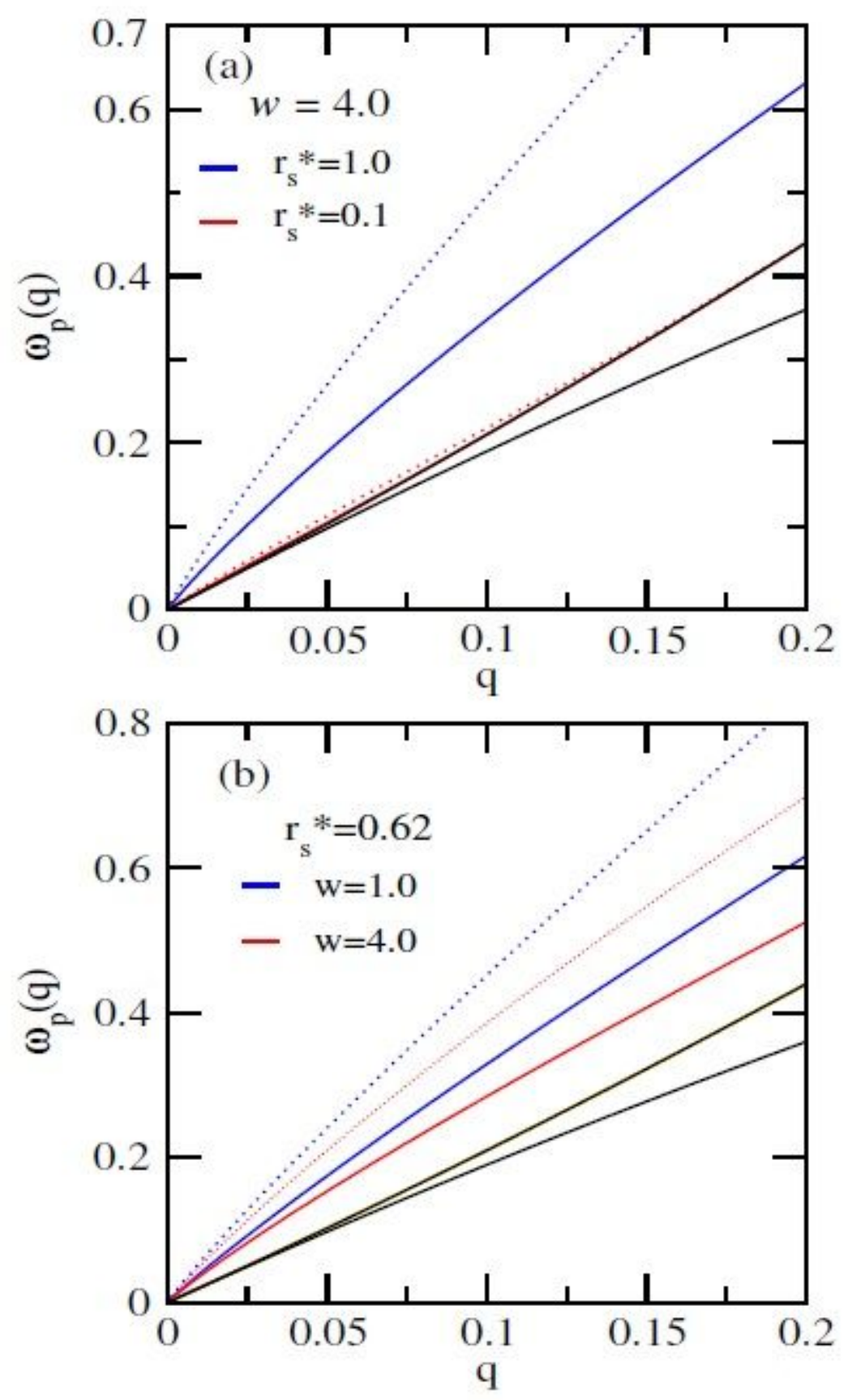

Figure 6

The dependence of ! $p(q)$ on $r^{*}$ s [in panel (a)] and $w$ [in panel (b)] at indicated wire parameters for gs $=1$ (solid lines) and gs $=2$ (dotted lines) in $\mathrm{HC}$ model. The thick black lines represent the boundaries of the single electron\{hole (eh) pair continuum at $r^{\star} s=0: 1$ [in panel (a)] and at $w=4: 0$ [in panel (b)]. 

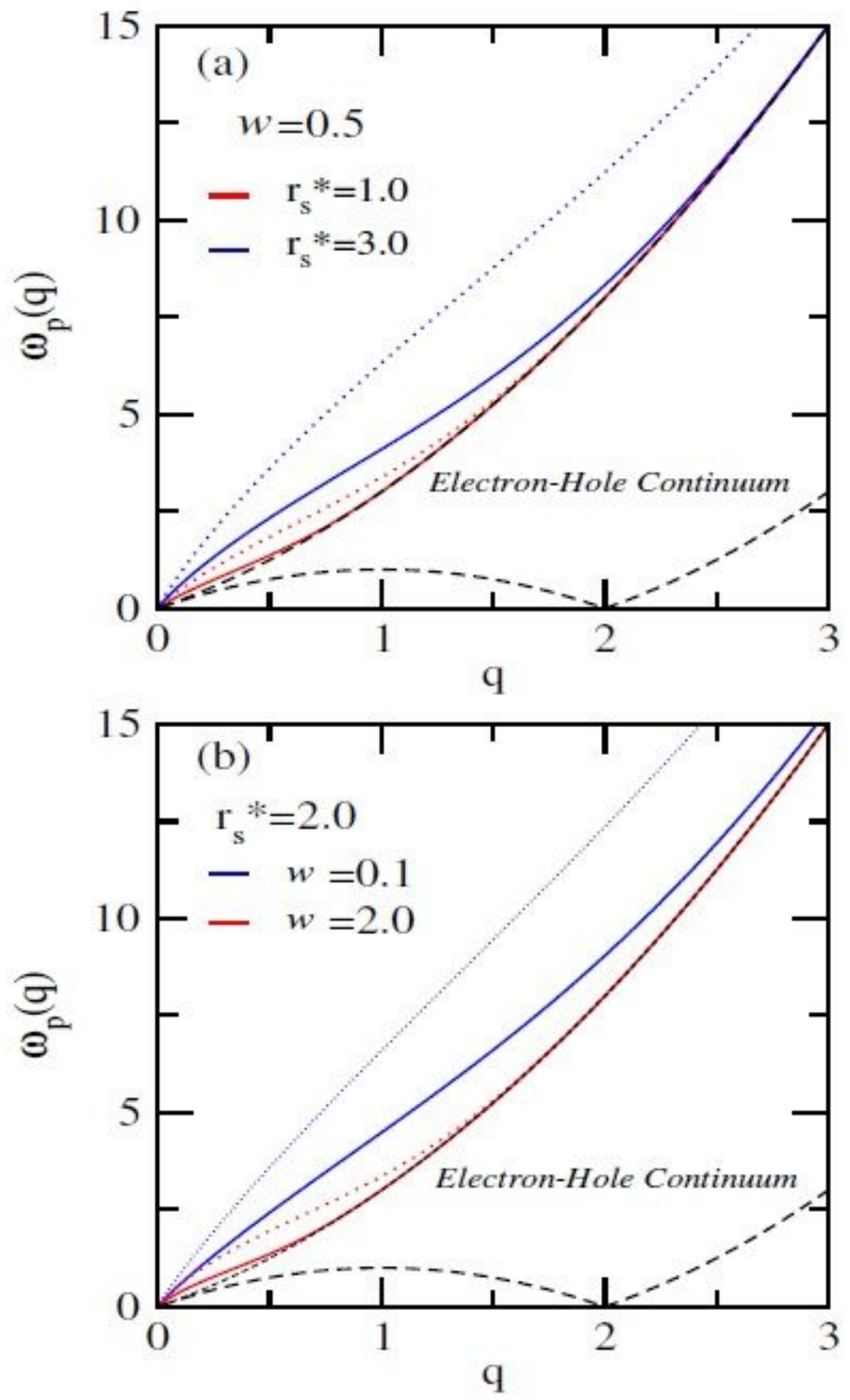

Figure 7

The dependence of $! p(q)$ on $q$ for indicated wire parameters at gs $=1$ (solid lines) and gs = 2 (dotted lines) in ISWC model. The thick dash\{dotted lines represent the boundaries of the single electron \{hole (eh) pair continuum at $r^{\star} s=1: 0[$ in panel (a)] and at $w=2: 0$ [in panel (b)]. 


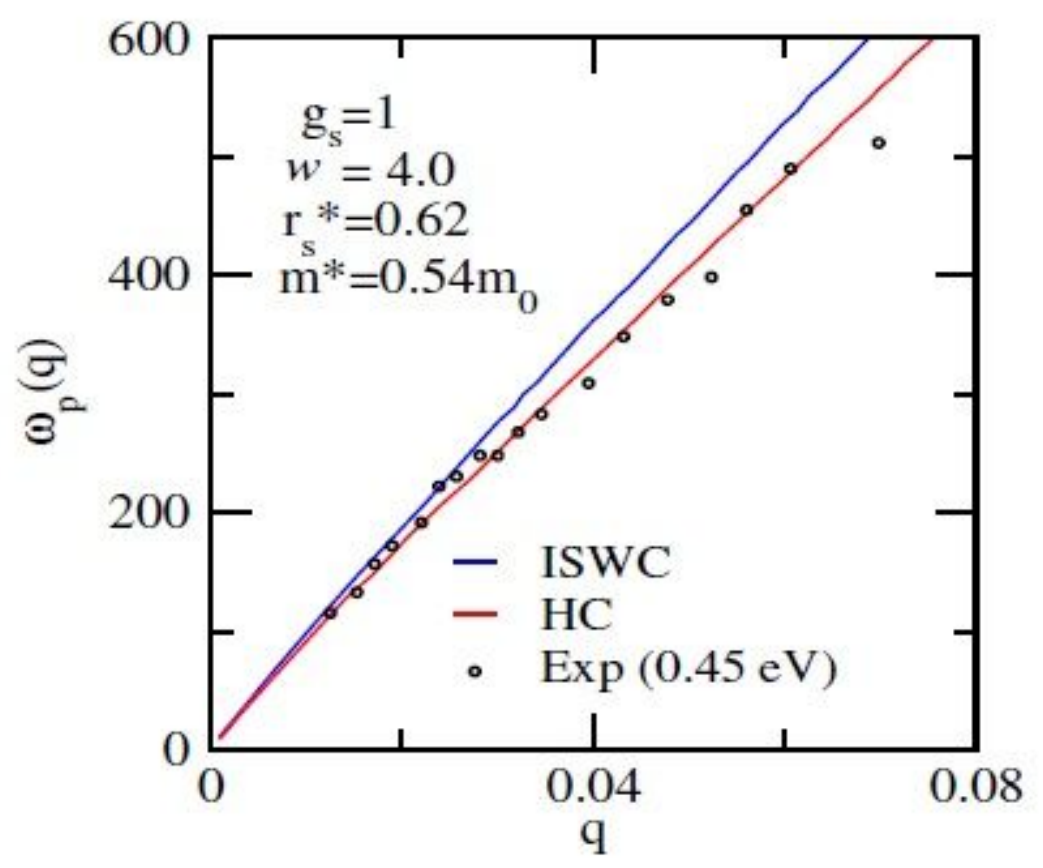

Figure 8

The variation of ! $\mathrm{p}(\mathrm{q})(\mathrm{meV})$ vs $\mathrm{q}$ at indicated wire parameters in $\mathrm{HC}$ and ISWC models. The symbols are the experimental data of Nagao et al. [11] for electron beams having incident energy of $45 \mathrm{eV}$.

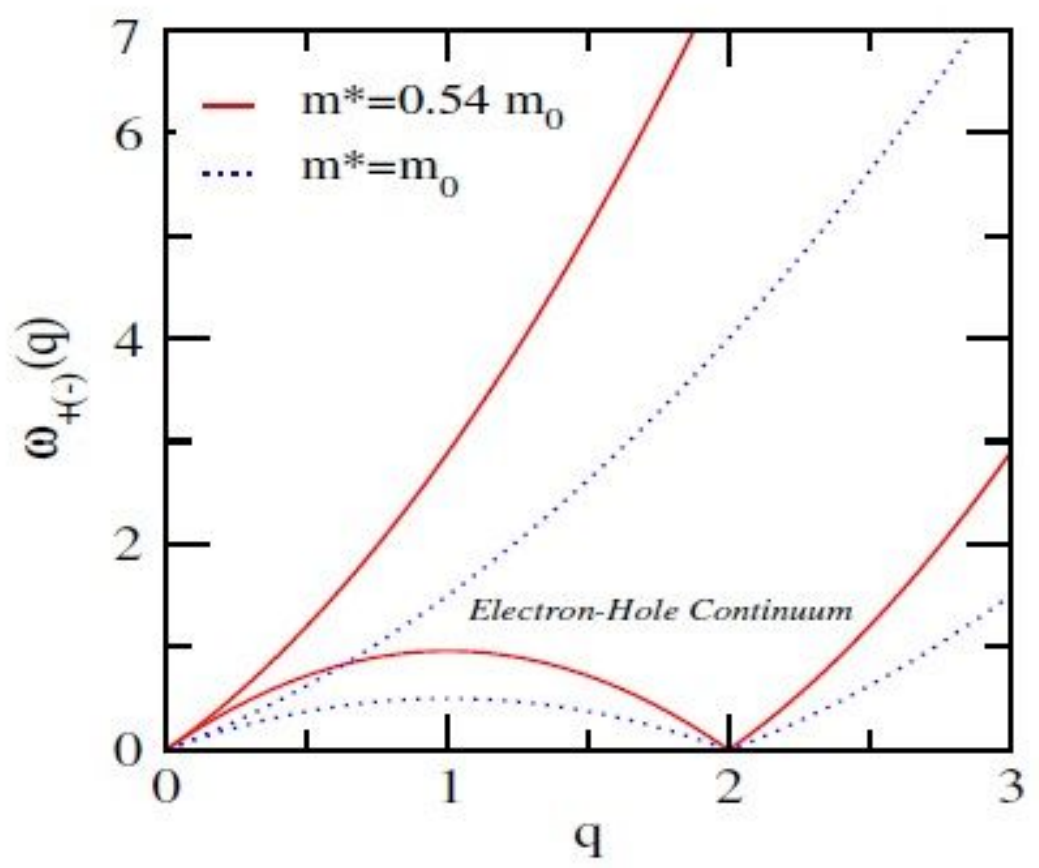

Figure 9

e-h pair continuum at $\mathrm{m}^{\star}=0: 54 \mathrm{~m} 0$ (red solid lines) and $\mathrm{m}^{*}=\mathrm{m} 0$ (blue dotted lines). 


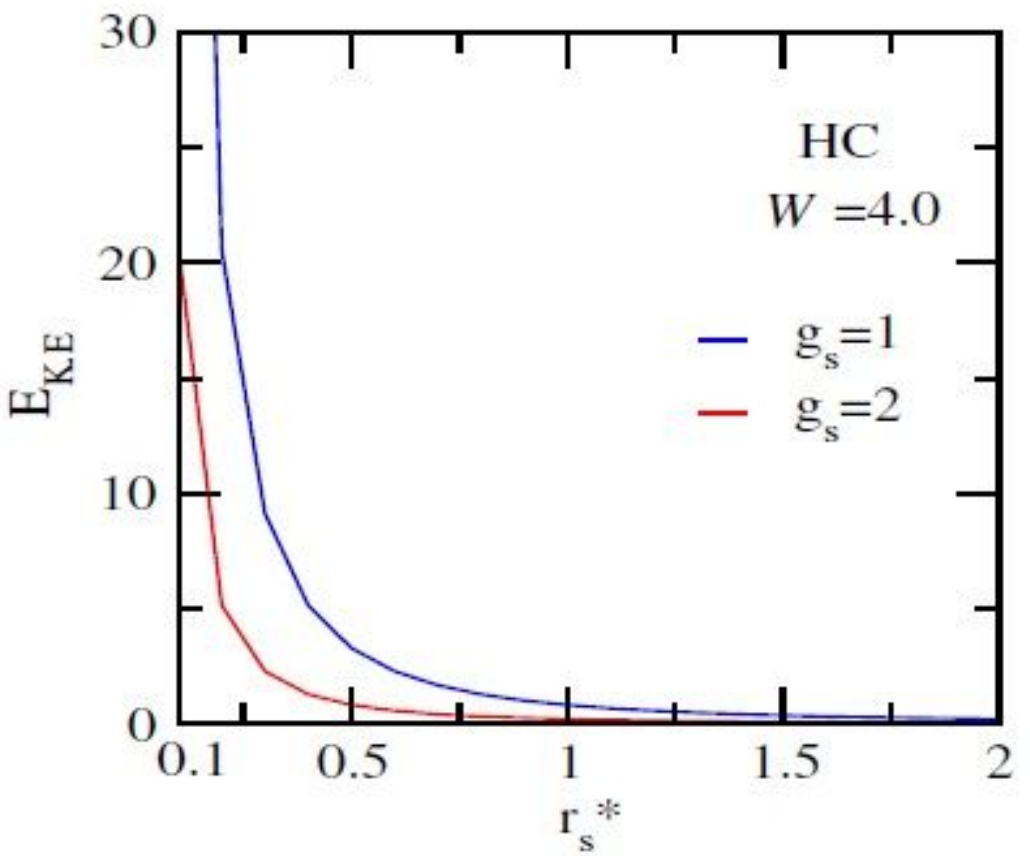

Figure 10

The $r^{\star}$ s-dependence of EK:E at w $=4$ in $\mathrm{HC}$ model. 

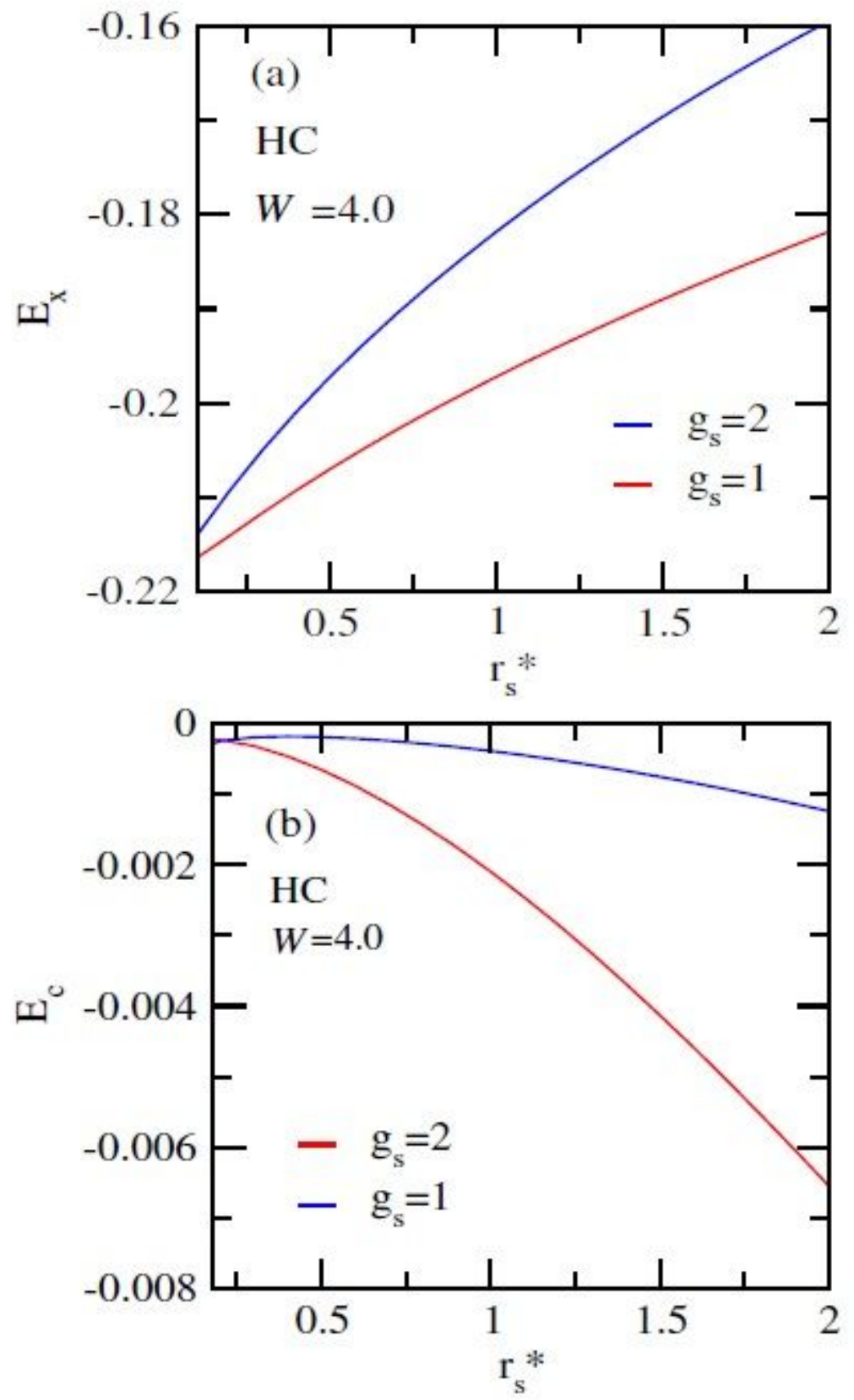

Figure 11

The r*s -dependence of Ex [in panel (a)] and Ec [in panel (b)] at w = 4 in $\mathrm{HC}$ model. 


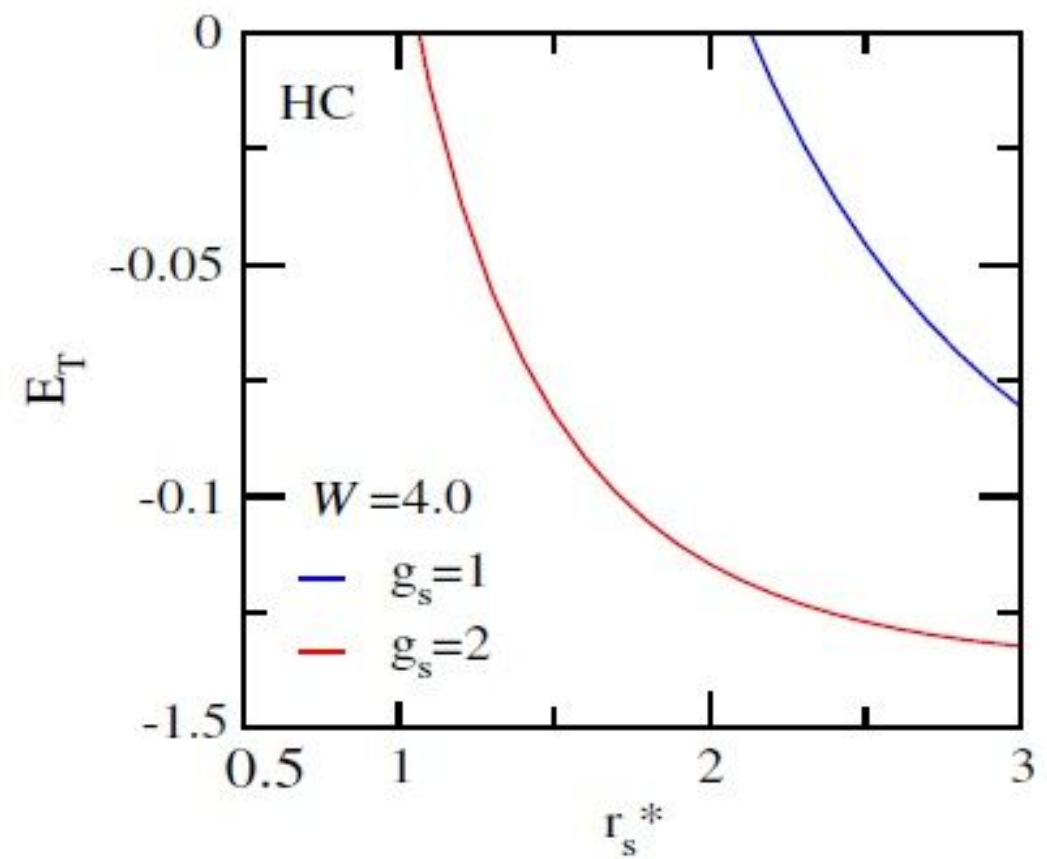

Figure 12

The $r^{\star}$ s -dependence of ET at $w=4$ in $\mathrm{HC}$ model. 